\title{
Review-Towards the Next Generation of Thermoelectric Materials: Tailoring Electronic and Phononic Properties of Nanomaterials
}

\author{
Olga Caballero-Calero ${ }^{\mathrm{a}, \mathrm{z}}$ and Roberto D'Agosta ${ }^{\mathrm{b}, \mathrm{c}, \mathrm{z}}$ \\ ${ }^{a}$ IMM-Instituto de Microelectrónica de Madrid (CNM-CSIC), E-28760 Tres Cantos, Madrid, Spain \\ ${ }^{b}$ Nano-Bio Spectroscopy Group and ETSF, Universidad del País Vasco, CFM CSIC-UPV/EHU, 20018 San Sebastián, \\ Spain \\ ${ }^{c}$ IKERBASQUE, Basque Foundation for Science, E-48013 Bilbao, Spain
}

In this review article we present the most relevant outcomes of the joint project "Tailoring Electronic and Phononic Properties of Nanomaterials: Towards Improved Thermoelectricity (nanoTHERM)", a Spanish research Consolider project focused on the understanding of the thermoelectric materials and the tailoring of both their electronic and phononic properties toward the optimization of the efficiency of thermoelectric devices working at low and high temperatures.

(C) The Author(s) 2017. Published by ECS. This is an open access article distributed under the terms of the Creative Commons Attribution 4.0 License (CC BY, http://creativecommons.org/licenses/by/4.0/), which permits unrestricted reuse of the work in any medium, provided the original work is properly cited. [DOI: 10.1149/2.0111703jss] All rights reserved.

(cc) BY

Manuscript submitted October 24, 2016; revised manuscript received December 29, 2016. Published January 24, 2017. This paper is part of the JSS Focus Issue on Thermoelectric Materials \& Devices: Phonon Engineering, Advanced Materials and Thermal Transport.

One of the most promising approaches to achieve high thermoelectric figures of merit has been the engineering of nanostructures or making use of nanostructure features in solid matter. The nanoTHERM project set out to investigate specific scientific questions concerning the nature of thermal transport at the nanoscale focusing in elemental materials, such as silicon, moving to highly complex polymer-based composites. We examine the role of dimensionality in the thermal conductivity down to length scale comparable to the typical wavelength for phonons, which depending on the system and thermal distribution, can be found in the range of nanometers. By varying the periodic modulation of matter as in phononic crystals we revealed the interplay of the wave and particle nature of phonons. The importance of the electronic band structure impacting the Seebeck coefficient that was little known has been investigated theoretically along with novel theoretical approaches to calculate the thermal conductivity. Since one of the initial aims was to enhance thermoelectric properties in the low temperature regime, we focused on polymerbased materials in order to understand the mechanisms at play in determining the electrical transport and finding ways to increase it since this was seen as the main drawback when considering practical applications. Among the most relevant results dealing with polymers as thermoelectric materials, we mentioned the obtained $Z T$ of 0.22 measured in PEDOT, which is one of the highest figures of merit obtained for polymers. In the case of PCDBT, its figure of merit has been enhanced by two orders of magnitude compared to the previously reported values, up to a $Z T$ of $2.6 \cdot 10^{-3}$ at room temperature.

On the other hand, for higher temperature thermoelectric materials our focus was primarily on typical and novel inorganic materials and structures from nanowires to multilayers, attempting to decouple thermal and electrical properties. The materials whose thermal properties we report here are the state-of-the-art. As an example, bismuth telluride nanowires, fabricated with different diameters, and measured, exhibited a measured reduction in the thermal conductivity when their diameter is reduced down to $0.5 \mathrm{~W} \cdot \mathrm{m}^{-1} \cdot \mathrm{K}^{-1}$. Moreover, the origin of the measured values has been understood thanks to the kinetic collective model developed in the collaborative work reported here framework of the project, namely. Another example is a full- CMOScompatible micro thermoelectric generator of $500 \mu \mathrm{m}^{2}$, based on silicon thin membranes, which provides an output of $4.5 \mu \mathrm{W} \cdot \mathrm{cm}^{-2}$ with a $5.5 \mathrm{~K}$ temperature difference.
Crucial to this collective work has been the harmonization of existing measurement and cross-calibration techniques together with the development of new ones. Thermal measurements at the nanoscale are known to be notoriously challenging and the results presented here have been obtained after a round robin exercise. Unfortunately, there is still a serious lack of metrology and concomitantly a lack of standards to measure thermal conductivity or conductance at the nanoscale.

With these developments in the measurement standardization, a wide variety of materials, organic, inorganic, homogeneous, composites, isotropic, etc. were characterized and optimized. A wide variety of techniques for materials synthesis were used, some existing, some newly developed.

It is worth mentioning that one of the main outcomes of this collaboration has been joint research combining theoretical and experimental groups and giving rise to extremely interesting results, which have pushed the frontier of knowledge in thermoelectric well beyond our initial expectations. One such collaboration was the development of the kinetic collective model, which explains the thermal conductivity reduction in the range of nanometers for different materials, matching successfully, for example, the experimental results obtained in bismuth telluride nanowires of different diameters.

This review article is organized in the following way. Firstly, the different materials investigated in the consortium, both organic (Nanoscale organic materials section) and inorganic (Nanoscale inorganic and hybrid materials section) will be presented, paying special attention to their preparation methods, the optimization routes followed in each case, and the influence of nanostructuring in their improved thermoelectric efficiency. In some cases, developments led to functional devices that will be also presented in this part. Secondly, the different experimental techniques developed within the consortium will be reviewed (Experimental techniques section). Thirdly, the theoretical models and developments, which have gone significantly further in thermal conductivity, size effects and properties prediction of different nanomaterials will be described (Theory section).

\section{Nanoscale Organic Materials}

Organic materials can be used for low temperature thermoelectric applications, where the non-toxicity, flexibility, and big area operation feasibility are required. They are easily manufactured, and the relatively low price and high abundance of their components is another important feature to be taken into account. Organic materials 
a)

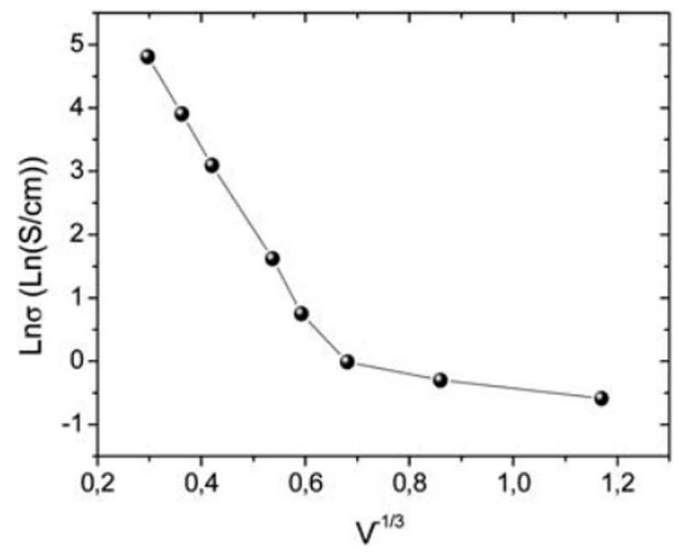

b)

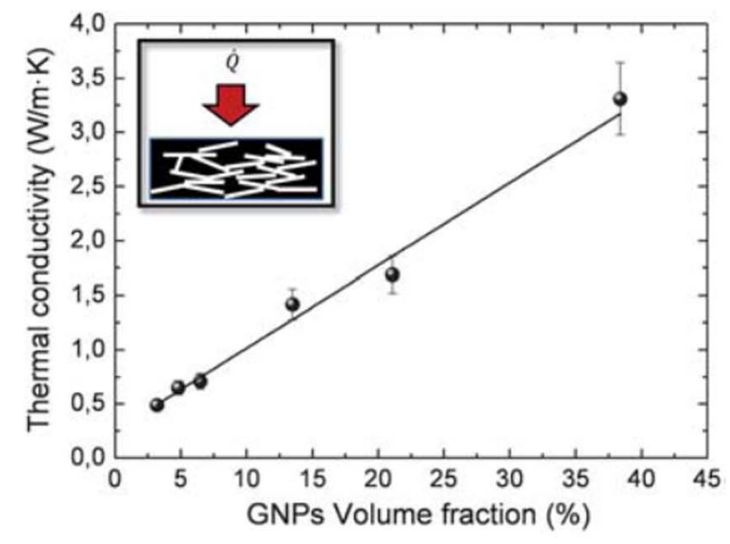

Figure 1. a) Plot of $\ln \sigma$ vs $V^{-1 / 3}$ showing a linear dependence, which reveals the tunnelling of the charge carriers. b) Linear fit of the thermal conductivity of the PANI as a function of the volume fraction of GNPs. The behavior can be described as a parallel thermal resistor (reproduced with permission from Ref. 14).

present a clear advantage over inorganic materials for thermoelectric applications, which is their low thermal conductivity. Nevertheless, their use in this field is restricted to intrinsically conducting polymers, given that a high electrical conductivity and high Seebeck coefficients are also required for efficient thermoelectric materials. Lately, a lot of research has been devoted to the tailoring of the doping and electrical properties of conducting polymers, along with the development of novel synthesized polymers with optimized electrical characteristics of both p- and n- type. This has triggered an important work from the theoretical point of view focused in understanding the transport properties of such materials (see for instance Reference 1 for a complete review).

In this review, we will present how among the many available conducting polymers, and after a careful screening process, the most suitable for further study were selected. Different approaches, such as wet techniques, ${ }^{2}$ blade-coating, ${ }^{3}$ electrochemistry, ${ }^{4}$ melting, ${ }^{5}$ etc. have been used to modify the doping, ${ }^{4}$ fabricate composites, ${ }^{6}$ and tailor other characteristics that have been studied for their optimization and to understand how nanostructuring of organic materials affects their thermoelectric performance. Then, two conductive polymers, namely Poly(3,4-ethylendioxitiophene) (PEDOT) and Poly(3hexylthiophene-2,5-diyl) (P3HT) will be described in detail, showing not only the advances obtained in their thermoelectric performance from a fundamental point of view, gaining a new insight into the behavior of such polymers, but also the implementation of device prototypes which show the potential of these materials.

Nanostructuring of semiconducting polymers.-The number of intrinsically conductive polymers and carbon based nanocomposites which were initially taken as potential targets for going a step further in the state-of-the-art in organic thermoelectric materials was huge: ${ }^{7}$ We explored from PEDOT with different additives or fillers prepared in thin film forms by electrodeposition, ${ }^{4} \mathrm{P} 3 \mathrm{HT}$ patterned via vapor printing $^{8}$ in order to modify its optical and electrical properties or mixed with both multi wall and single wall carbon nanotubes (CNTs), ${ }^{9}$ polycarbonatediol polyurethane with expanded graphite, ${ }^{2,6}$ vitreous carbon, ${ }^{10}$ to micro and nanofibers of carbon grown by vapor liquid solid (VLS) techniques. ${ }^{11-13}$

For instance, in the case of polyaniline (PANI), initially a thorough analysis of the synthetic factors that affect its thermoelectric properties was performed. Therefore, the polymerization kinetics, structural characterization, and thermoelectric properties were studied. ${ }^{14}$ Then, different approaches were taken in order to obtain both $\mathrm{p}$ - and n-type polymers, such as preparing nanocomposites with optimized thermoelectric efficiency by mixing PANI with Graphene Nanoplates (GNP). ${ }^{14}$ In order to increase the electrical conductivity of these nanocomposites, electrical conductive fillers GNP were also intro- duced in the composite, obtaining power factors of $14 \mu \mathrm{W} \cdot \mathrm{m}^{-1} \cdot \mathrm{K}^{-1}$ when $50 \%$ wt GNPs were added, ${ }^{14}$ which is three orders of magnitude with respect to PANI itself (see Figure 1, where the effect of the charge carriers in the electrical conductivity is shown (Figure 1a) while the thermal conductivity (Figure 1b) is maintained low).

The effect of nanostructuring on conductive polymers was also studied in the case of (Poly[N-9'-heptadecanyl-2,7.-carbazole-alt-5,5(4'7'-di-2-thienyl-2',2',3'-benzothiadiazole)] (PCDTBT). The thermal conductivity of film PCDTBT doped with $\mathrm{FeCl}_{3}$ to increase the electrical conductivity was measured with both the $3 \omega$-SThM (Scanning Thermal Microscope working in $3 \omega$ mode) and the photoacoustic techniques, obtaining around $1 \mathrm{~W} \cdot \mathrm{m}^{-1} \cdot \mathrm{K}^{-1}$ at optimal $\mathrm{FeCl}_{3}$ doping, resulting in a $Z T$ of $2.6 \cdot 10^{-3}$ at room temperature, which is two orders of magnitude larger than the best reported for such polymers, ${ }^{15}$ showing the potential of proper polymer nanostructuring.

Poly(3,4-ethylendioxitiophene), PEDOT.-PEDOT is a semimetallic or semiconductive polymer, which has been prepared via evaporation and powder solution, with good reproducibility, also with polystyrenesulfonate (PEDOT:PSS). The composite formed with PEDOT:PSS and different contents of graphite was studied as thermoelectric material for room-temperature applications, showing power factors over $5 \mu \mathrm{W} \cdot \mathrm{m}^{-1} \cdot \mathrm{K}^{-1}$ for composites with a $80 \%$ wt of expanded graphite. ${ }^{16}$ Nevertheless, different films prepared through electrochemical polymerization on gold surfaces have been prepared, controlling the oxidation level ${ }^{17}$ or tuning its doping, giving rise to PEDOT: $\mathrm{ClO}_{4}$, PEDOT:PF , and PEDOT:BTFMSI (bis(trifluoromethylsulfonyl)imide). In the last case, an increase in the thermoelectric efficiency up to a maximum of $Z T$ of 0.22 at room temperature was obtained, which is among the highest values found in literature for polymers where the actual reported state-of-the-art is $Z T=0.4$, although there are not many organic systems that achieve $Z T$ values over $0.1 .^{4}$ In addition, the doping level can also be optimized by an electrochemical process. ${ }^{17}$ Finally, using these PEDOT polymeric materials two electronic devices have been developed. A thermoelectric module using materials obtained by one-step electrochemical synthesis was fabricated in the form of 16 large aspect ratio ( $2 \mathrm{~mm}$ wide times $80 \mathrm{~mm}$ long) parallel stripes deposited on flexible substrates. The maximum power output was $25 \mathrm{nW} .^{18}$ The thermal sensor developed was based on an optimized PEDOT nanofilm (120 $\mathrm{nm}$ in thickness) integrated into an electronic circuit, showing its performance by switching on a LED with the temperature increment produced by a fingertip ${ }^{18}$ (See Figure 2).

Poly(3-hexylthiophene-2,5-diyl) P3HT.-P3HT is a conjugate polymer in which the orientation of the polymer chains can be easily controlled $^{3}$ and can form stable composites when mixed, for instance, 
a)

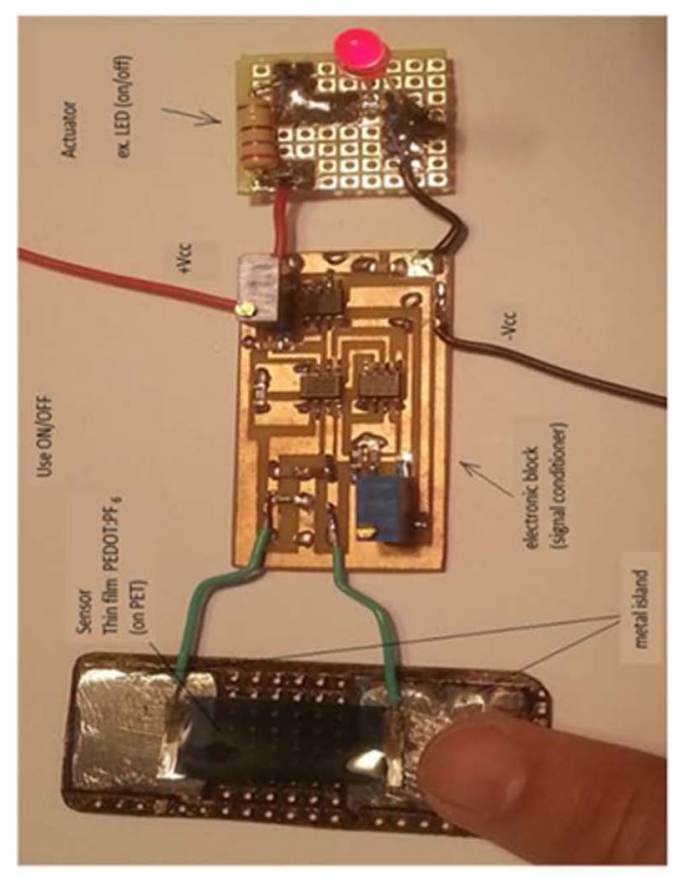

b)
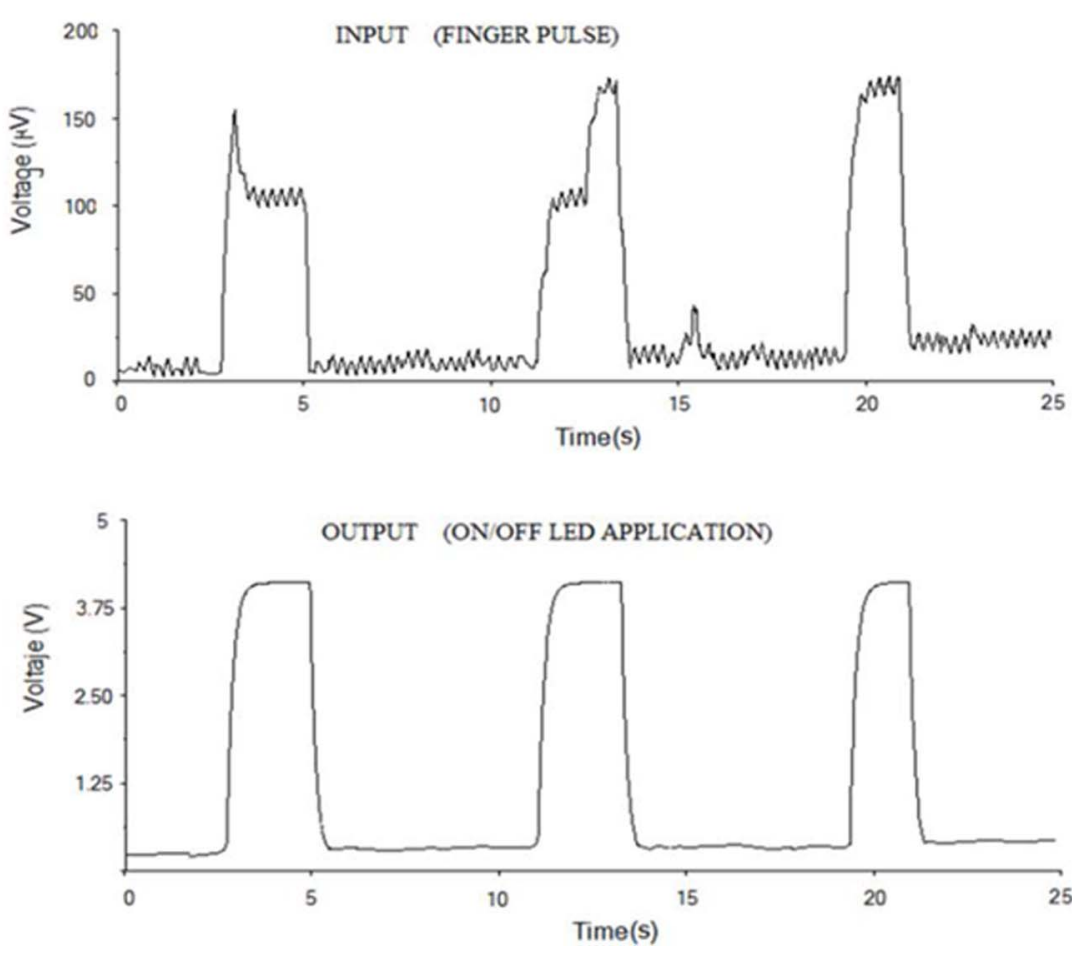

Figure 2. a) Picture of the actual sensor device presented in Ref. 18. b) voltage output generated by a finger pressing the thermal polymer sensor (reproduced with permission from Ref. 18).

with fullerenes. ${ }^{19} \mathrm{P} 3 \mathrm{HT}$ has attracted attention from the thermoelectric community because there were different approaches suggesting that by optimizing the p-doping or mixing it with CNTs it will be competitive with other thermoelectric polymers. Nevertheless, the obtained power factors were too small and showed that a new approach was necessary. A new route was based on combining the p-doping optimization and the fabrication of a composite with CNTs. Therefore, P3HT polymer matrices filled with CNTs were fabricated in such a way that they form thick films on flexible substrates (such as poly(ethylene terephthalate), (PET). ${ }^{9}$ The measured power factor for these composites reached a maximum value of around $95 \mu \mathrm{W} \cdot \mathrm{m}^{-1} \cdot \mathrm{K}^{-2}$ for P3HT/42-81\% single wall CNT. Moreover, n-type CNTs and p-type CNTs mixed with P3HT gave rise to p-type and n-type films, with a change in the Seebeck coefficient from positive $700 \mu \mathrm{V} \cdot \mathrm{K}^{-1}$ to $-8 \mu \mathrm{V} \cdot \mathrm{K}^{-1} .{ }^{20}$ Finally, under UV illumination the $\mathrm{p}$ - to n-type behavior can also be changed (See Figure $3 a$, where a scheme of the device-fabrication based change in the Seebeck coefficient from positive values (p-type) to negative values (n-type) under UV illumination is shown). These concepts were implemented in a proof of concept flexible organic thermoelectric generator ${ }^{20}$ (See Figures $3 b$ and $3 c$ ). Also, a semi-empirical phenomenological model has been developed to understand the thermal transport properties of these P3HT/CNTs composites, which will be discussed in Polymeric materials section.

One dimensional nanometric structures of P3HT have also been fabricated through infiltration inside anodic aluminum oxide (AAO) templates. The fabrication of nanowires of different diameters (from 300 to $15 \mathrm{~nm}$ ) led to the first proof of how the confinement in the nanoscale leads to changes in the crystallinity of the polymer. The study of the photoluminescence, structural characterization, and scanning electron microscopy of the different nanowires, showed that in the case of melt-processed nanowires the two dimensional confinement influences the orientation of the polymer crystals. ${ }^{5}$ Moreover, the decrease of the thermal conductivity with decreasing diameter, measured with a $3 \omega-$ SThM (see Figure 4 ), showed a correlation between the size confinement effects in polymers and their thermal transport, which can be related with the increasing presence of crystals oriented in the [100] direction parallel to the nanowire length when decreasing the diameter ${ }^{21}$ (see Figure 4).

\section{Nanoscale Inorganic and Hybrid Materials}

Since the discovery of the thermoelectric effect, the most outstanding materials as far as performance is concerned, have been inorganic semiconductors, from chalcogenides, ${ }^{22}$ such as $\mathrm{Bi}_{2} \mathrm{Te}_{3}$, for room-temperature applications, to silicides, ${ }^{23}$ which are optimal for a)

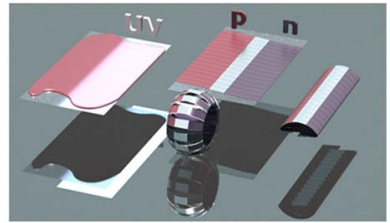

b)

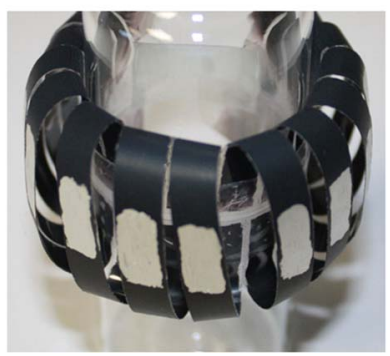

c)

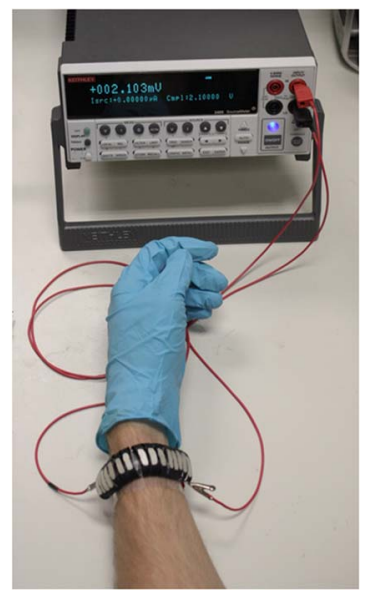

Figure 3. a) Seebeck coefficient of P3HT composite with $20 \%$ CNTs (triangles) and 30\% CNTs after different times of UV irradiation, where it can be seen that the composites turn from p-type to n-type. b) proposed fabrication steps of the implementation of a device which takes advantage of the p-type, ntype conversion, and images of the actual device (reproduced with permission from Ref. 20) 
a)
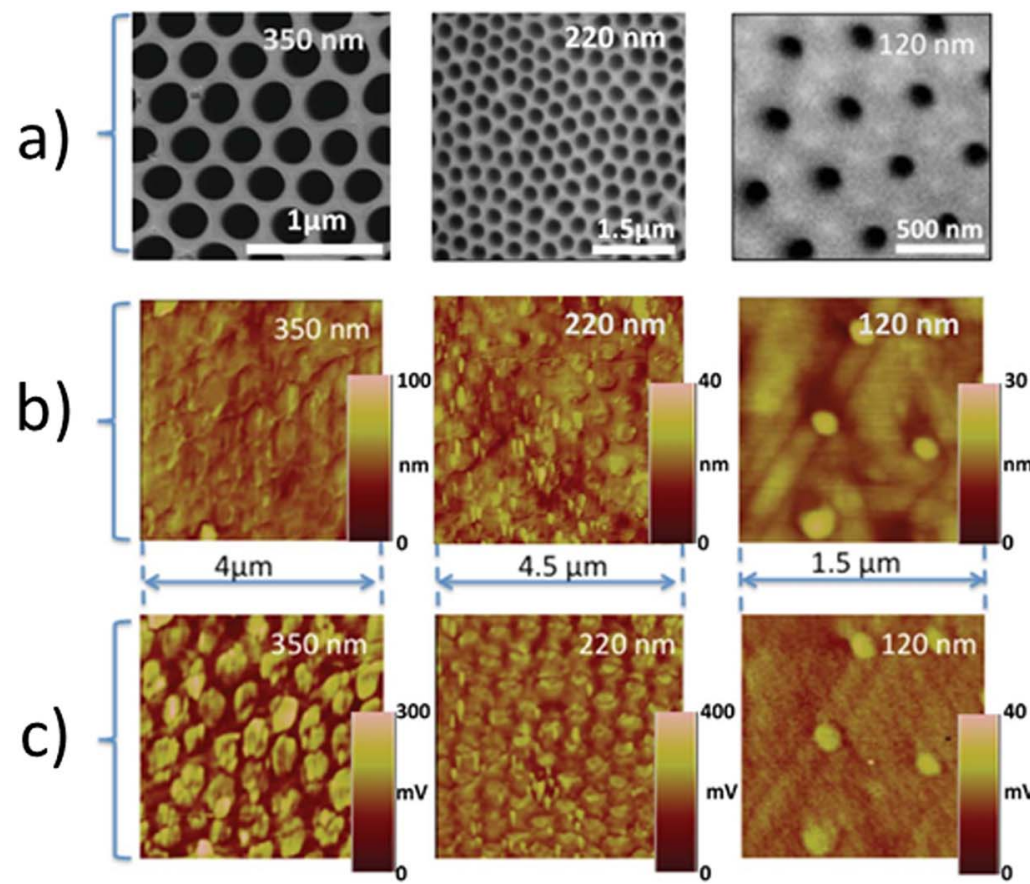

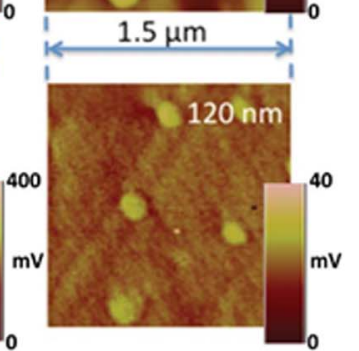

d)

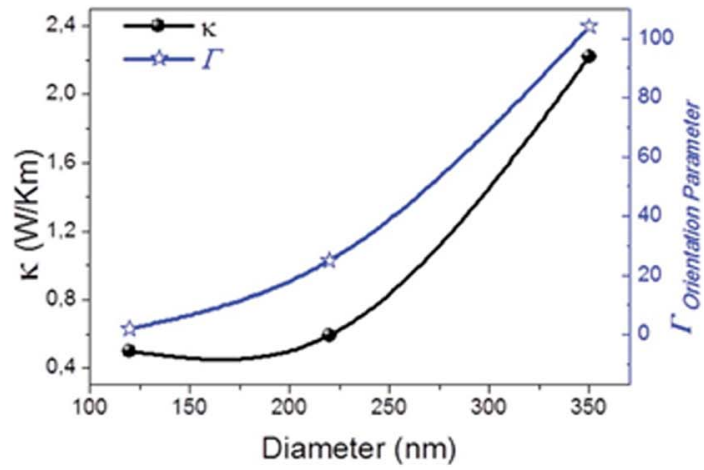

Figure 4. a) SEM pictures of the different diameter alumina matrices, b) topographic images of the same matrices filled with P3HT nanowires c) SThM images of the same nanowire arrays. d) measured thermal conductivity (black circles) and the orientation parameter (stars) of P3HT nanowires as a function of their diameter, being the orientation parameter defined as the relation between the areas of the (100) peak in the XRD diffractogram in the directions perpendicular and parallel to the nanowire axis times a certain coefficient (reproduced with permission from Ref. 21).

high temperature applications, mainly $\mathrm{SiGe}$, which is the leading thermoelectric material at this range. In the case of inorganic materials, their main limitation lies in their thermal conductivity, which is generally high and should be reduced in order to improve their thermoelectric efficiency. Nevertheless, a reduction in the thermal conductivity is normally associated to a decrease in the electrical conductivity, which as a whole makes the total efficiency decrease. One way of increase the thermoelectric performance of these materials is to engineer their structure at the nanoscale in order to tailor the thermal conductivity of the lattice. This can be achieved through different routes, for instance, by developing superlattices of different materials, ${ }^{24}$ nanowires, ${ }^{25,26}$ phononic crystals with an increased phonon dispersion, ${ }^{27}$ or ultra-thin membranes. ${ }^{28}$

Multilayer quantum dot superlattices (QDSL).-Molecular beam epitaxy (MBE) techniques are especially suitable for the fabrication of nanostructures with a high control over the stoichiometry and size of the desired design. This technique has been used to grow germanium quantum dots and quantum dot superlattices embedded in a silicon matrix. ${ }^{29,30}$ Theoretical results on these structures were also carried out, showing how the strain in these kinds of nanostructures would lead to carrier confinement ${ }^{31}$ and even the valence bands could be engineered, producing electron-like or hole-like bands depending on the thickness of the quantum wells, composition and hydrostatic pressure. ${ }^{30}$ This strained structures also showed carrier trapping in the germanium quantum dots (see Figure 5), obtaining a way of enhancing the Seebeck coefficient. ${ }^{24}$

Electrodeposited nanowires of chalcogenide materials $\left(\mathrm{Bi}_{2} \mathrm{Te}_{3}\right.$ and $\boldsymbol{S b _ { 2 }} \boldsymbol{T e}_{3}$ ).-Chalcogenides are among the most widely used thermoelectric materials for applications around room temperature. Although they have been widely studied, there is a lack of deep understanding of their thermoelectric properties when grown via electrochemical deposition, showing a poorer performance than when grown by other methods. Nevertheless, electrochemistry is a cost-effective method that allows the fabrication of different structures by the use of templates. Therefore, the growth of films with this technique and the optimization of its different parameters was performed, ${ }^{32}$ obtaining films with $33 \%$ increase in the Seebeck coefficient with respect to other $\mathrm{Bi}_{2} \mathrm{Te}_{3}$ electrochemically grown films, even $60 \%$ for $\mathrm{Bi}_{2} \mathrm{Te}_{2-\mathrm{y}} \mathrm{Se}_{\mathrm{y}}{ }^{33,34}$ Then, to study how nanostructuring affects its thermoelectric behavior, assisted template deposition of n-type bismuth telluride inside porous alumina templates was performed, ${ }^{35}$ giving rise to bismuth telluride nanowire arrays with diameters in the order of tens of nanometers and lengths in the order of micrometers. In this aspect, a new development on alumina templates with really small pores allowed the fabrication of the smallest diameter nanowires to that point, with diameters of $25 \mathrm{~nm}$ and $15 \mathrm{~nm} .{ }^{36}$ The measurement of the thermal properties on these nanowires posed also an experimental challenge, and finally a $3 \omega-\mathrm{SThM}$ measurement of the nanowires inside the AAO matrix was performed, obtaining a reduction in the thermal conductivity for nanowires of $200 \mathrm{~nm}$ in diameter from $2.2 \mathrm{~W} \cdot \mathrm{m}^{-1} \cdot \mathrm{K}^{-1}$, which is the

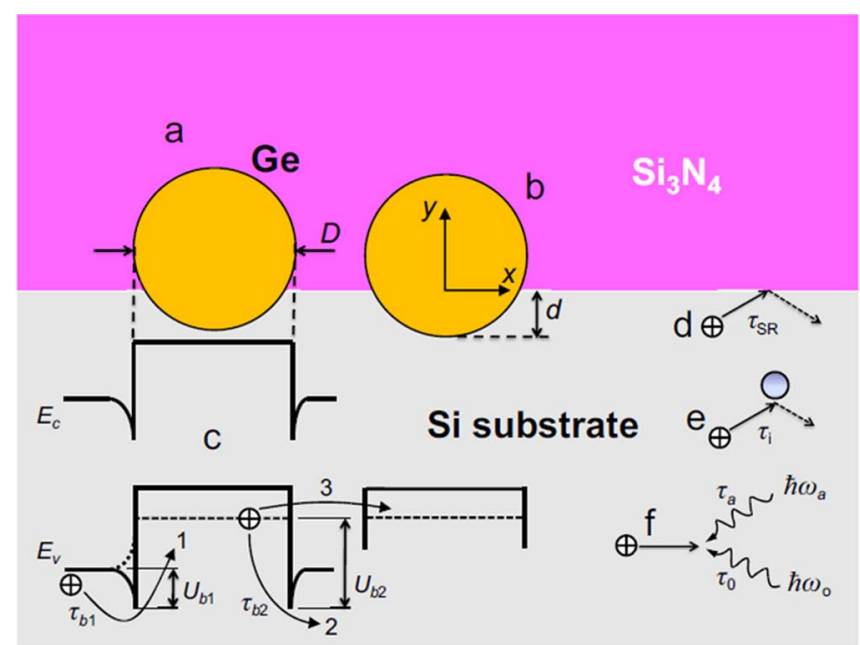

Figure 5. Scheme of the geometry of the structure with the conduction bands and band edge alignments, demonstrating the trapping of holes (reproduced with permission from Ref. 24). 

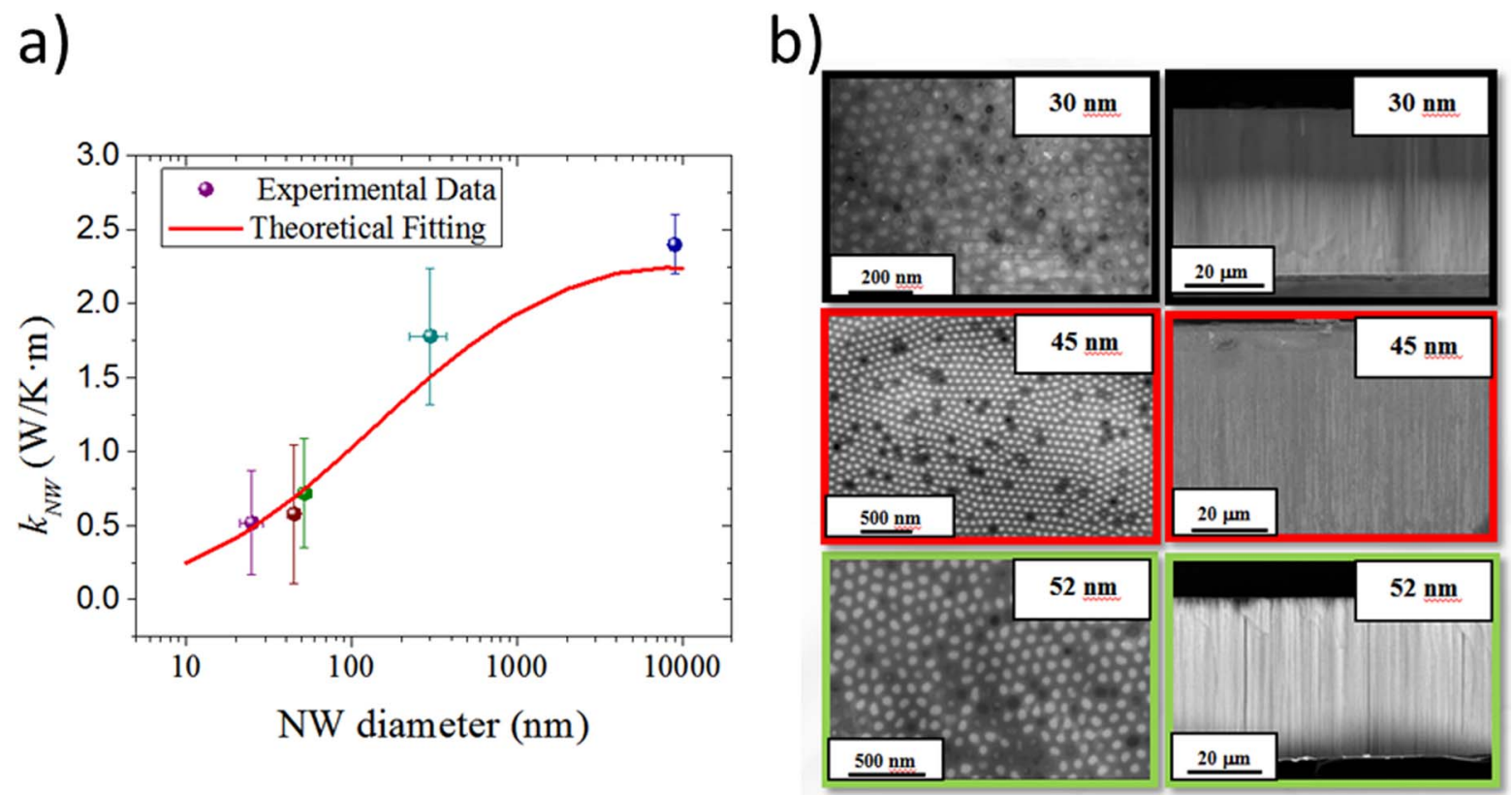

Figure 6. Right experimental measurement of the thermal conductivity of different $\mathrm{Bi}_{2} \mathrm{Te}_{3}$ nanowires as a function of their diameters (spheres) and the theoretical model derived from the Kinetic-Collective theory. Left, SEM images of the top and lateral view of some of these nanowires (reproduced with permission from Ref. 34).

value for bulk, to around $1.4 \mathrm{~W} \cdot \mathrm{m}^{-1} \cdot \mathrm{K}^{-1} \cdot{ }^{37}$ A step forward to prove the reduction of the thermal conductivity due to the size confinement has been done thanks to a joint collaboration between experiments performed at the Microelectronics group in Madrid and the theory developed by the Universidad Autonoma de Barcelona. On the one hand, the first group grew, via electrochemical deposition, nanowires of different diameters (from 300 down to $25 \mathrm{~nm}$ ) all with the same orientation (110), and the same composition, $\mathrm{Bi}_{2} \mathrm{Te}_{3}$. Then, the thermal conductivity of these nanowires was measured with two different techniques, namely photoacoustic and SThM, obtaining decreasing values down to $0.5 \mathrm{~W} \cdot \mathrm{m}^{-1} \cdot \mathrm{K}^{-1}$ for the nanowires of $30 \mathrm{~nm}$ in diameter. On the other hand, the theoretical group predicted, using the kinetic-collective model (KCM, See Kinetic collective model section), the behavior of the thermal conductivity for these nanowires, being the reduction in this parameter caused mainly by the alteration of the mean free path of the acoustic phonons due to size confinement. The agreement between theory and experiment is quite good (see Figure 6), and this work gives us a better insight on the actual phenomena that take place at the nanoscale. ${ }^{32}$

Graded structures.-These structures were initially proposed theoretically as a way of demonstrating a thermal rectification effect that was derived from the KCM (see Kinetic collective model section). The basis of this effect is that the theory shows that the thermal boundary resistance (TBR) of an abrupt $\mathrm{Si}_{1-\mathrm{x}} \mathrm{Ge}_{\mathrm{x}} / \mathrm{Si}$ interface is quite different depending on the direction of the heat flow. The asymmetry of the structure has such a remarkable effect on the heat flow propagation, that a difference of around the $40 \%$ is expected, depending on the heat flow direction. To test this thermal rectification effect, multilayered samples of graded SiGe sequences were fabricated via MBE on silicon substrates. Different studies on the influence of alloy inhomogeneities on the composition or strain of these layers, ${ }^{38}$ showed that the transport properties of such structures could be tailored, obtaining thermal conductivity values as low as $2.2 \mathrm{~W} \cdot \mathrm{m}^{-1} \cdot \mathrm{K}^{-1}$. 39

Silicon thermoelectrics: suspended membranes and phononic crystals.-In the case of silicon thin layers, the theory on the dispersion relations in ultrathin silicon membranes ${ }^{40}$ showed a great potential for their use in thermoelectricity. Experimentally, free standing silicon membranes with thicknesses down to $6 \mathrm{~nm}$ were fabricated in such a way that the strain was controlled and then they were measured with Raman Thermometry to study the thermal conductivity, which was reduced from the bulk value $\left(148 \mathrm{~W} \cdot \mathrm{m}^{-1} \cdot \mathrm{K}^{-1}\right.$ at room temperature) with decreasing the membrane thickness, with a minimum value for membranes of $9 \mathrm{~nm}$ thickness of $9 \pm 2 \mathrm{~W} \cdot \mathrm{m}^{-1} \cdot \mathrm{K}^{-1}$, due to the increased phonon scattering at the surfaces, ${ }^{41}$ with a great influence of the surface on its properties. ${ }^{42}$ In the case of suspended layers of less than $20 \mathrm{~nm}$, their in-plane thermal conductivity was also measured with an special custom setup to measure suspended structures in a certain microchip design, obtaining values of $19 \mathrm{~W} \cdot \mathrm{m}^{-1} \cdot \mathrm{K}^{-1}, 43$ which could be then cut with gallium ions by focused ion beam (FIB). This fabrication step produces a further decrease of the thermal conductivity, due to the partial amorphization of the silicon, which can be later recovered (in a polycrystalline form) with appropriate annealing treatments.

A further step with this micro device is to fabricate a micro thermoelectric generator in which the active thermoelectric material is the thin silicon suspended membrane, which can be tuned from ntype to p-type with appropriate dopants. The first prototype of such generator (with a suspended membrane of $500 \times 500 \mu \mathrm{m}^{2}$ ) gave a power output of $4.5 \mu \mathrm{W} / \mathrm{cm}^{2}$ under a $5.5 \mathrm{~K}$ temperature difference, ${ }^{44}$ and it is fully compatible with CMOS technologies. Similar silicon membranes were also studied with asynchronous optical sampling to investigate the decay times of the confined coherent phonons. ${ }^{45}$

Other structures, which are quite appealing for thermoelectricity and that have been produced on silicon, are phononic crystals (PnCs). These PnCs have been proposed for the control of heat at the nanoscale. The periodically modulated elastic properties of these structures lead to strong modifications of the phonon dispersion and therefore to the phonon group velocity and lifetime, which can directly affect the phonon propagation. Thus, heat transport is expected to be influenced by the modified dispersion relation when the wavelength of the phonons involved in the heat transport is commensurate to the characteristic sizes of the phononic crystal.

We have exploited present fabrication techniques to demonstrate the modification of the dispersion relation of both surface, ${ }^{46}$ and plate acoustic phonons $s^{27,47}$ at the hypersound. Although the roomtemperature thermal conductivity of plate-based PnCs should not be affected by coherent scattering of phonons, we applied the two-laser Raman thermometry (see Two-laser Raman thermometry section) to 

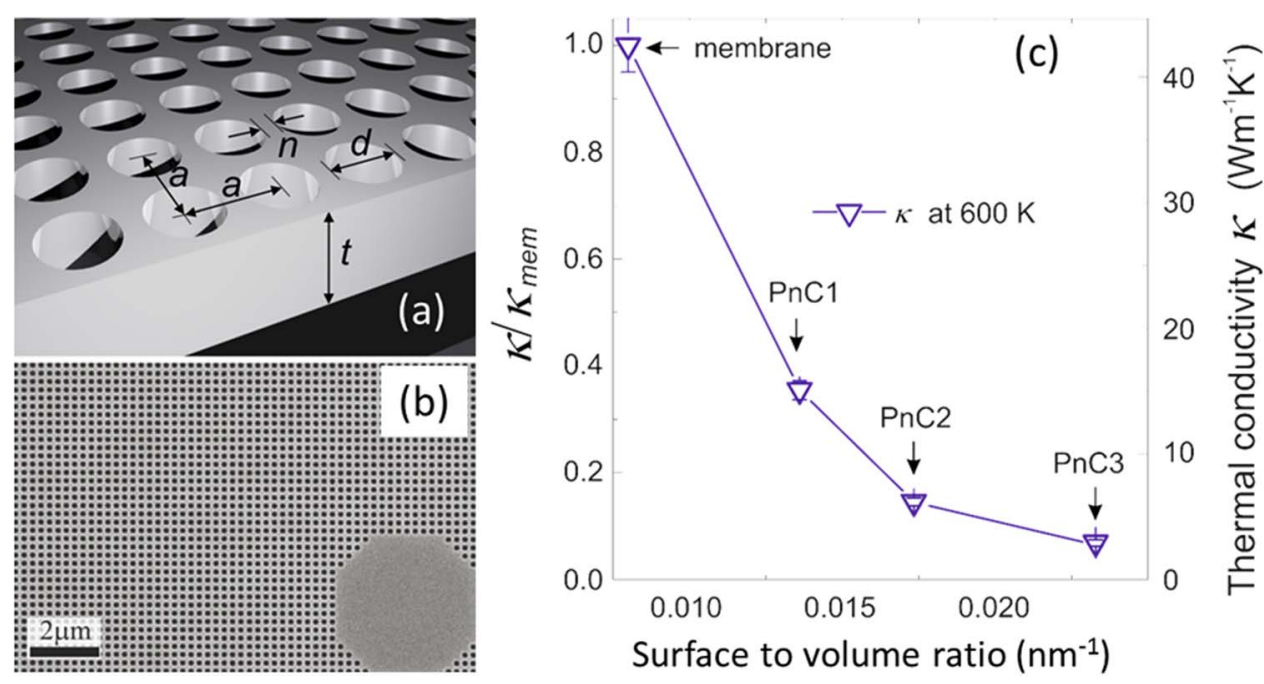

Figure 7. a) Schematic of the periodically patterned membrane. b) SEM image of a sample with $a=250 \mathrm{~nm}$ and $d=140 \mathrm{~nm}$. c) Intrinsic $\kappa$ as a function of the surface to volume ratio.

study the in-plane thermal conductivity and its temperature evolution from room temperature up to about $1000 \mathrm{~K} \cdot{ }^{48}$ Starting from $\mathrm{t}$ $=250 \mathrm{~nm}$ thick single crystalline silicon (001) membranes, three samples labeled $\mathrm{PnC} 1, \mathrm{PnC} 2$ and $\mathrm{PnC} 3$ of an intentionally similar hole diameter of about $d=135 \mathrm{~nm}$ and differing in the lattice constant, $a$, of 300, 250 and $200 \mathrm{~nm}$, respectively (see Figures $7 \mathrm{a}$ and $7 \mathrm{~b})$ were studied. The thermal conductivity $\kappa$ is found to be reduced to values close to a-Si with increasing surface to volume ratio ${ }^{46}$ (see Figure $7 \mathrm{c}$ ). The thermal conductivity for $\mathrm{PnC} 3$ is reduced 40 times with respect the Si bulk value at $300 \mathrm{~K}$. Such reduction can only be achieved for thicknesses down to $10 \mathrm{~nm}$ in un-patterned membranes. Moreover, it has been seen that the temperature evolution of the thermal conductivity, $\kappa(\mathrm{T})$, can be effectively tuned and approaching to a regime where $\kappa$ is almost insensitive to T. We have addressed the question to what extend disorder influences the phononic properties of $2 \mathrm{D}$ phononic crystals in the hypersound and thermal range, both experimentally ${ }^{49}$ and theoretically. ${ }^{27,46}$ The introduction of disorder in the PnCs drastically modifies the hypersonic phonon spectrum resulting in the suppression of coherent acoustic phonon modes. Measurements of the thermal conductivity have shown a same amount of the suppression of the thermal conductivity for both ordered and disordered samples with respect to the un-patterned membrane. The results reveal the impact of surface roughness and disorder on the observation of coherent effects in phononic crystals and demonstrate that the room-temperature thermal conductivity in comparable phononic crystals should not be affected by the change of the phonon dispersion resulting from coherent boundary scattering. The reduction of the inplane thermal conductivity is mainly determined by the shortening of the phonon mean free path due to the diffusive scattering of phonons at the boundaries of the PnCs.

Finally, from the allow SiGe, nanomeshes have been fabricated via DC sputtering on highly ordered porous alumina templates. The grown $\mathrm{Si}_{0.8} \mathrm{Ge}_{0.2}$ film replicated the structure of the alumina, and the growth technique, along with the type of alumina template, provides a high control over the geometry of the obtained nano-mesh. These structures showed thermal conductivity values as low as $0.55 \mathrm{~W} \cdot \mathrm{m}^{-1} \cdot \mathrm{K}^{-1}$. These large area and scalable thermoelectric materials maintain the power factor of the bulk, but with reduced thermal conductivity. ${ }^{23}$

In summary, Si nanostructures, either ordered or disordered, can be designed in such a way that they exhibit reduced thermal conductivity, ${ }^{50}$ or even produce thermal rectification ${ }^{51}$ in nanoporous silicon (even in inhomogeneous media ${ }^{52}$ ).

STO thin films and multilayers.- $\mathrm{Nb}: \mathrm{SrTiO}_{3}$ (STO) is a nontoxic and thermally stable material which is considered for high- temperature thermoelectric applications due to its electronic properties leading to high Seebeck coefficients and, consequently, high power factors provided the thermal conductivity is kept small. The nature of the orbitals contributing to the electronic band structure and to the density of states close to the Fermi energy are usually invoked to explain the high Seebeck coefficients of up to about 600 $\mu \mathrm{V} / \mathrm{K}$. This augurs well for STO as a thermoelectric material and formed the background to the research undertaken based on thin films grown by pulsed laser deposition (PLD) on $\mathrm{LaAlO}_{3}$ (LAO) and other substrates, with substitutional $\mathrm{Nb}$ doping from 2 to $20 \%$ in thin films of various thickness (few tens of nm to hundreds of $\mathrm{nm}$ ) and multilayers. Electrical and thermal measurements were made at various temperatures leading to the selection of $\mathrm{SrTiO}_{3} \mathrm{Nb}_{0.02}$ grown on $\left(\mathrm{LaAlO}_{3}\right)_{0.3}\left(\mathrm{Sr}_{2} \mathrm{TaAlO}_{6}\right)_{0.7}$ (also known as LSAT) and on $\mathrm{MgO}$ to show the most promising values of $Z T$. These results are undergoing comparison to electronic band structure calculations as there is an obvious link to the enhanced Seebeck coefficient of Nb-doped STO thin films. In this kind of perovskite-like ceramics, the formation of a twodimensional electron gas depends very much on the kind of surface and interface structure of the two materials in contact (substrate and thin film). ${ }^{53}$ Moreover, depending on the growth conditions there may be ordered grains ${ }^{54}$ and elongated columnar structures below $10 \mathrm{~nm}$, which contribute to the complexity of the data simulation. The data from STO studies is still undergoing further analysis via theoretical models. ${ }^{55}$ To date the most promising results are obtained in a thin film of $2 \%-\mathrm{Nb}$-doped STO grown on LSAT, with a carrier concentration of $8.37 \times 10^{20} \mathrm{~cm}^{-3}$, carrier mobility of $5.4 \mathrm{~cm}^{2} / \mathrm{Vs}$, electrical conductivity of $723.5 \mathrm{~S} / \mathrm{cm}$, Seebeck coefficient of $-320 \pm 15 \mu \mathrm{V} / \mathrm{K}$, and a thermal conductivity of $2.70 \pm 0.01 \mathrm{~W} / \mathrm{mK}$, yielding a ZT of $0.8 \pm 0.1$, where all values at room temperature. This value compares very well to ZT at $1000 \mathrm{~K}$ of 0.37 obtained in single crystal and polycrystalline ceramics ${ }^{56,57}$ and the largest ZT obtained in a superlattice of $\mathrm{SrTiO}_{3} / \mathrm{Nb}: \mathrm{SrTiO}_{3}$, namely, 2.4 at $300 \mathrm{~K}^{58}$

Other nanostructured inorganic materials.-Given that nanostructuring seems the best way of reducing the thermal conductivity of inorganic materials and thus improve their thermoelectric performance, different approaches were undertaken to obtain nanostructures (thin films and nanowires) of different materials, which could be then measured and optimized.

On the one hand, one-dimensional structures, such as nanowires, were developed in different materials, apart from those discussed earlier in the text. It has been shown theoretically that some properties of the materials, such as thermal conductivity, can be tuned by shaping them in nanowires, with influence of both the size and the shape, ${ }^{59}$ and 


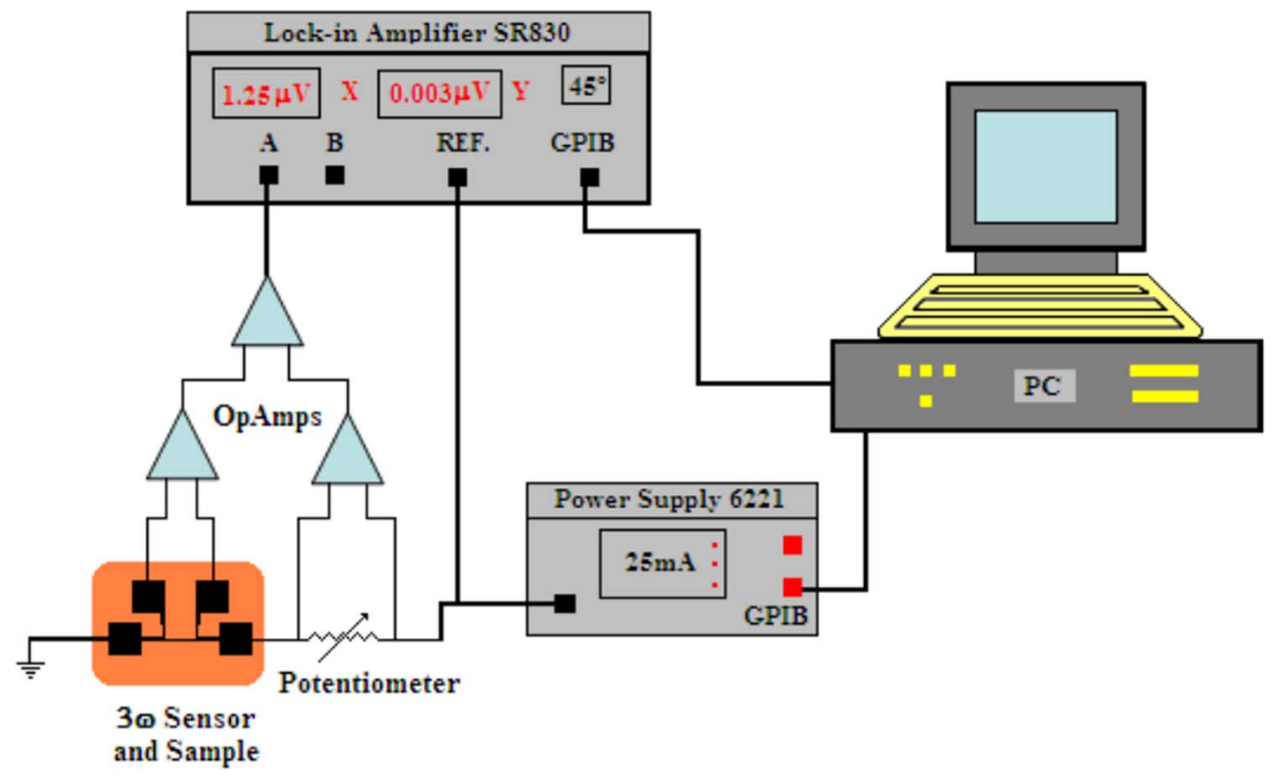

Figure 8. Schematic of the circuit for a $3 \omega$ measurement.

also from the experimental point of view a great effort has been done to characterize those structures. ${ }^{60}$ For instance, perovskite nanowires $\left(\mathrm{La}_{1-\mathrm{x}} \mathrm{Ca}_{\mathrm{x}} \mathrm{MnO}_{3}\right.$ and $\left.\mathrm{Nd}_{1-\mathrm{x}} \mathrm{Ca}_{\mathrm{x}} \mathrm{CoO}\right)$ fabricated via hydrothermal techniques. Their drawback was that, although they exhibited reasonable Seebeck coefficients, their electrical conductivity was low and gave low thermoelectric efficiency. ${ }^{7,61}$ Other types of nanowires were, for instance, those developed by a novel method that gave rise to ordered arrays of germanium nanowires patterned by FIB deposition of gold seed catalysts on silicon substrates, ${ }^{62}$ combining VLS methods with molecular-beam epitaxy (MBE) growth. These nanowires show variations at the nanoscale in the composition and strain, which were characterized by tip enhanced micro-Raman. ${ }^{63}$ Another widely studied thermoelectric material, namely $\mathrm{ZnO}$, was also prepared in the form of nanowires grown by VLS. ${ }^{64}$ Finally, silicon and germanium nanowires were also prepared. These structures were proposed to study the behavior of the thermal conductivity in nanowires, as well as in-plane $\mathrm{SiGe}$ alloy nanowires grown by MBE on Si substrates ${ }^{39}$ with control over the composition. ${ }^{39}$

On the other hand, two-dimensional superlattice structures of nonstoichiometric $\mathrm{SiO}_{\mathrm{x}}$ containing $\mathrm{Si}$ nanoparticles with diameters between 1.5 and $2.5 \mathrm{~nm}$ were also fabricated with a two-step procedure which consisted of the electron beam evaporation of a silicon layer on a germanium or glass substrate, with an oxygen partial pressure constant throughout the process to guarantee the growth of suboxides in the film. These amorphous and porous layers were then annealed between $450^{\circ} \mathrm{C}-700^{\circ} \mathrm{C}$ under argon atmosphere. ${ }^{65}$ Actually, the study of the photoluminescence of these structures at high hydrostatic pressures from both the theoretical and the experimental point of view is a sign of their quantum confinement. ${ }^{65}$ Other two-dimensional nanostructures studied in the framework of their thermoelectric performance were $\mathrm{Cr} / \mathrm{V}_{2} \mathrm{O}_{5}$ thin films (with $\mathrm{ZT}$ of 0.16 at room temperature ${ }^{66}$ ), $\mathrm{Al}: \mathrm{ZnO}$ sputtered thin films (with a $Z T$ over 0.1 , three times bigger than previously reported values ${ }^{67}$ ), silicon thin layers and graphene membranes, both from the theoretical ${ }^{28,40,68}$ and experimental ${ }^{43}$ point of view.

\section{Experimental Techniques}

Apart from the usual techniques used for thermoelectric characterization of materials and devices, given the wide variety of materials and configurations (bulk, 2D thin films, 1D nanowires, and even OD quantum dots), there has been a great effort in these years to develop techniques, which could measure all of them in a reasonable way. Commercial or well-established experimental systems have been used, such as four-probes for electrical conductivity, microchips with resistors for both acting as heaters and temperature measurement for Seebeck and thermal conductivity measurements, steady-state systems for thermal conductivity measurements of bulk samples, tipenhanced Raman scattering, etc. In this section we will not describe all of them, although most of the used systems are not commercial ones and have been developed in the framework of the project. Instead, we will describe in detail two systems that have been improved and developed specifically addressing the challenges of performing measurements that could not be done with such precision or in the configurations that can be addressed with them. Initially, we will describe the $3 \omega$-method, which has been used for the measurement of extremely thin suspended membranes, as well as, in combination with a modified atomic force microscope, to measure individual nanowires. Secondly, the two-laser-Raman-thermometry, which was specifically developed to study the thermal properties of suspended membranes, with a high resolution for in-plane measurements, which are usually not available with other techniques.

The measure of the thermal conductivity is usually a complex experimental problem lacking at the moment a proper standard. This implies that common measurements of the same sample might lead to different results, if the techniques used are somewhat not compatible. Well aware of these problems, in the consortium we have developed novel measurement techniques, properly suited for the common materials used, and we have validated our results via round-robin measurements of some common thermoelectric device. In particular, this second step allows us to have the necessary confidence on the uniformity of the results in the different groups of the consortium for a better comparison of the progress made in each material.

$3 \omega$ method.-The $3 \omega$ method $^{60}$ relies on the injection of a sinusoidal current using a metallic transducer (heater/current sensor) and the measurement of the third harmonic $(3 \omega)$ voltage $\left(V_{3 \omega}\right)$ through a lock-in amplifier (see Figure 8 for a description of the system developed by one of the partners).

One can prove that $V_{3 \omega}$ is related to the temperature difference across the material and the thermal conductivity can be obtained by the appropriate solution of heat transfer equations ( $\mathrm{se}^{60}$ and reference therein). The setups developed in the consortium can reliably measure the thermal conductivity of bulk, thin films and suspended membranes between 4 and $350 \mathrm{~K}$. The $3 \omega$ method is ideally suited to measure thermal conductivity of thin film insulating materials with 
(a)

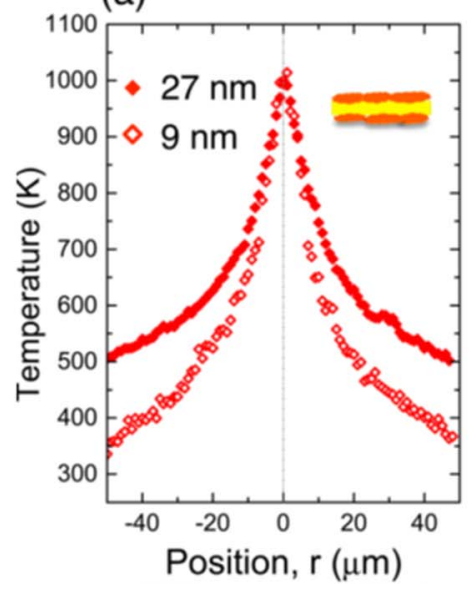

(b)

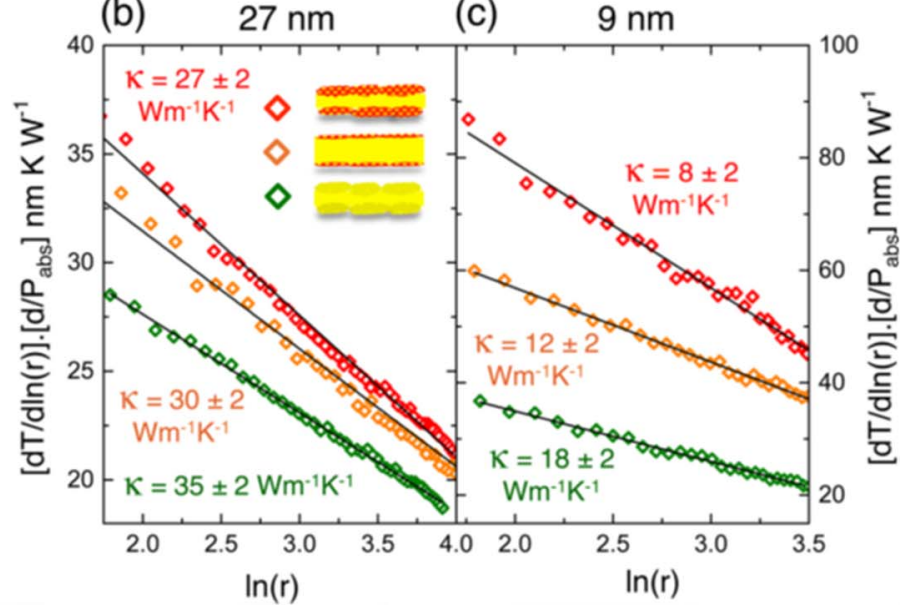

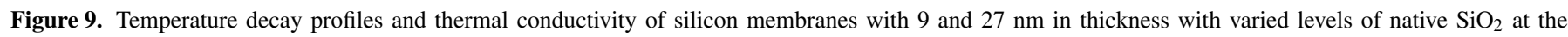

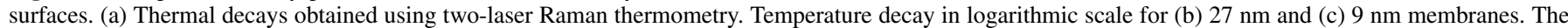

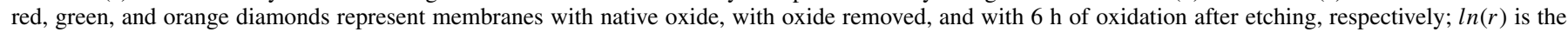
logarithm of the distance $r$ from the center (reproduced with permission from Ref. 42).

low thermal conductivities. In this case the temperature gradient that originates within the film of interest is large enough to easily detect the $3 \omega$ component of the in-phase voltage. In most cases a differential measurement with a reference sample is performed to eliminate the contribution of the substrate and precisely determine the conductivity of the film. In situations where the substrate has a much higher thermal conductivity than the film, the film is relatively thick and the sensor width is narrow $(5-10 \mu \mathrm{m})$, the contribution of the substrate to the overall $\Delta T$ can be safely neglected. This method can also be applied to electrically conductive materials introducing an insulating layer between the layer and the metallic heater/sensor. This layer should be included in the reference sample to eliminate its contribution to the temperature difference. In rough samples this is very problematic since the thickness of the insulating layer needs to be high and that increases the error in the evaluation of the thermal conductivity of the active layer.

As a limitation of the method, we can name lithography, which is normally required to define the transducer on the surface of the sample under study. Therefore this procedure is not applicable to polymers or organic materials. However, with the advances in mechanical tooling now it is possible to fabricate shadow masks with the right dimensions to directly grow the metallic heater/sensor on the sample surface with the appropriate shape avoiding wet chemical process. Nevertheless, this technique has been successfully applied to the measurement of the thermal conductance of suspended structures, both membranes and nanowires, with a working temperature between 4 and $400 \mathrm{~K}$, with control at the $\mathrm{mK}$ level.

The $3 \omega$ method can be also extended to the measurement of the thermal properties of nanowires embedded in a matrix (like for example the $\mathrm{Bi}_{2} \mathrm{Te}_{3}$ in the alumina matrix) when combined with a Scanning Thermal Microscope $(3 \omega-\mathrm{SThM})(60)$. This technique provides a step forward in the characterization of nanostructures since it is the only available capable of measuring the thermal conductivity of single NWs embedded in a matrix as well as the thermal conductivity of the composite locally. In principle, the technique is more versatile and straightforward than other methods. In the consortium this method has been used to measure, for the first time, the thermal properties of individual nanowires of a semiconducting polymer inside an alumina matrix. We observe a reduction of thermal conductivity, from $\lambda_{\mathrm{NW}}=$ $2.29 \pm 0.15 \mathrm{~W} \mathrm{~K}^{-1} \mathrm{~m}^{-1}$ to $\lambda_{\mathrm{NW}}=0.5 \pm 0.24 \mathrm{~W} \mathrm{~K}^{-1} \mathrm{~m}^{-1}$, when the diameter of nanowires is reduced from $350 \mathrm{~nm}$ to $120 \mathrm{~nm}$, which correlates with the polymer crystal orientation measured by WAXS. ${ }^{21}$

Two-laser raman thermometry.-This technique is based on the use of two lasers, one for heating the sample, and the other to measure the actual temperature of the sample at different distances from the center of the heating laser. It is rooted in the Raman Thermometry, where a laser is focused at the center of a suspended membrane, producing local heat due to the absorption of this incident radiation, which is reflected in a red-shift of certain optical phonons of the material. The extension of this technique to obtain a thermal field map is based on using a second laser to probe the local temperature through the spectral position of a Raman active mode, which will be shifted depending on the actual temperature. This system can have a spatial resolution of $500 \mathrm{~nm}$ and can measure temperatures with an accuracy of $\pm 2 \mathrm{~K}$. An example of such a measurement can be found in Figure 9. Another advantage of this technique is that it is contactless and needs no further preparation of the sample under investigation. It has been tested in the measurement of free standing single crystalline $\mathrm{Si}$ membranes of thicknesses from $250 \mathrm{~nm}$ to $2 \mathrm{~nm}^{42}$

\section{Theory}

State-of-the-art in transport at the nanoscale.-The consistent progress made over the years by scientists on the ability of design, create, and measuring small electronic devices have pushed an equal progress in the development of the theoretical tools to understand the experimental results. In a few occasions, the theory even anticipated the experiments: an emblematic case being the prediction of the quantum of electrical conductance made by R. Landauer with his theory of quantum transport in low dimensional system and then verified years later in beautiful experiments. Nowadays, the Landauer approach, ${ }^{69}$ coupled with the advances in the electronic and phonon band structure calculations, is one of the standard tools for obtaining numerical prediction on the electronic and thermal transport coefficients for nanoscale systems. In other fields, for example in the calculations of the thermal conductance associated with phonon transport, progress has been made both in improving the models and in building new theories for a better description of the atomic structures. In this review, we will discuss the advancements made within the nanoTHERM consortium on the different theoretical aspects of the electrical and thermal transport at the nanoscale. We will briefly introduce the relevant theories to define the state-of-the-art and then we will discuss the progress made in the different fields. As a closing remark, an interesting problem we are facing in the theory of thermoelectric devices is how to reconcile the results of the diverse approaches available, especially at the interface between quantum and classical mechanics. Finally, we point out that there is the need of alternative theories to validate the current tools. ${ }^{70}$ 
The Landauer approach.-A widespread physically intuitive approach to the calculation of the conductance in nano- and mesoscopic systems is the Landauer's formula: One assumes that the fine details of the system are irrelevant far from the structure under investigation in some part of space dubbed "reservoir". The only requirement being that the reservoirs are opaque with respect to electron tunneling, i.e., an electron entering a reservoir cannot leave it. Therefore the electron conductance is the sum over all the possible leads, of the probability of an electron to leave a leads and to enter another. ${ }^{69}$ Fixed bias and temperature gradient can be applied to keep the reservoirs polarized. The device is then connected to the reservoirs by "leads" that go smoothly from the former to the latter. In this structure, the transport characteristics are determined solely by the probability amplitude for one electron to come out of a reservoir, cross the lead-device-lead region, and be absorbed by one of the other reservoirs. One can show that the probability amplitude for crossing the device can be related with the electronic structure of the device itself. One of the reasons why the Landauer approach has become a standard is in the advancement made in electronic structure calculations mainly driven by the success of Density Functional Theory (DFT). Nowadays, therefore many people do refer to the Landauer-Buttikker-DFT (LB-DFT) approach to quantum transport.

In the LB-DFT, we start from the linear out-of-equilibrium Onsager's relations for the charge $(j)$ and heat $\left(j_{h}\right)$ currents flowing along the device,

$$
\begin{aligned}
& j=L_{00} \Delta \mu+L_{01} \Delta T \\
& j_{h}=L_{10} \Delta \mu+L_{11} \Delta T
\end{aligned}
$$

LB-DFT approach to thermal transport allows to expressing the Onsager's coefficients $L$ 's in terms of the transmission amplitude. In linear response with respect to the thermal gradient $\Delta T$ and the polarization potential $\Delta \mu$, one obtains the general expressions:

$$
L_{i j}(\mu, T)=\int_{-\infty}^{\infty} d E(E-\mu)^{i+j} \frac{\partial f(E, T, \mu)}{\partial E} \mathcal{T}(E) .
$$

Here, the chemical potential is defined as $\mu=\left(\mu_{R}+\mu_{L}\right) / 2$, where $\mu_{R}$ and $\mu_{L}$ are the chemical potentials of the reservoirs, respectively. By assumption, $\mu_{L}-\mu_{R}=\Delta \mu \ll \mu_{L}, \mu_{R}$. The same is true for the temperature $T=\left(T_{R}+T_{L}\right) / 2$, with $\Delta T=T_{L}-T_{R} \ll T_{R}, T_{L}$, and $f(E, T, \mu)$ is the Fermi function. We assume indeed that the reservoirs are large and at equilibrium at given temperature and chemical potential. Moreover, it is assumed that the electrons in the reservoir do not interact. This last assumption is justified by the fact that in metal the screening length is small. Finally, $\mathcal{T}(E)$ is the transmission probability for one electron with given energy $E$ to travel across the device. The theory needs to calculate this quantity to make the set of equations of any use.

Given the Onsager's relations, we obtain the following relations between the microscopic parameters that enter the figure-of-merit and the integral $L$ 's,

$$
\begin{aligned}
\sigma & =e^{2} L_{00}(\mu, T), \\
S & =\frac{L_{10}(\mu, T)}{e T L_{00}(\mu, T)}, \\
\kappa_{e} & =\frac{1}{T}\left(L_{11}(\mu, T)-\frac{L_{10}^{2}(\mu, T)}{L_{00}(\mu, T)}\right) .
\end{aligned}
$$

Here, $\kappa_{e}$ is the electronic contribution to the thermal conductance, defined by

$$
\kappa_{e} T=\left.\frac{j_{h}}{\Delta T}\right|_{j=0} .
$$

We have assumed implicitly that we can write the total thermal conductance as $\kappa=\kappa_{e}+\kappa_{l}$, where $\kappa_{l}$ is the crystal (either phononic or vibronic) contribution. This is valid if the electron-phonon interaction is negligible.
One can formulate a LB-DFT theory also for phonon transport. In doing so, we obtain the following expression for the thermal conductance $\kappa_{l}$ in linear response in terms of the transmission probability of a phonon across the device,

$$
\kappa_{l}=\int_{0}^{\infty} d \omega \omega^{2} \frac{\partial n(\omega, T)}{\partial T} \tau(\omega),
$$

where $n(\omega, T)$ is the Bose distribution of non-interacting phonon at given temperature $T$, and $\tau(\omega)$ is the probability that one phonon with energy $\omega$ could cross the device. The fact that one can tackle on the same ground both the vibrational and electronic contribution to the figure-of-merit is one of the greatest advantages of this theory. Moreover, its simplicity in expressing all the quantities of interest in terms of the transmission probability and this latter in term of a microscopic model of the device, is invaluable in making the theory predictive.

Boltzmann transport equation.- - Another theoretical approach that is routinely used in the calculation of the transport coefficients is based on the Boltzmann equation. ${ }^{69}$ This is a semi-classical approach in the sense that we define a distribution function in the phase space $\{\boldsymbol{q}, \boldsymbol{p}\}$ of all particles, $f(\boldsymbol{p}, \boldsymbol{q}, t)$. Here $\boldsymbol{q}$ and $\boldsymbol{p}$ are vectors representing the positions and momenta of all the particles, and $t$ is the time. The number of particles in a small volume $d \boldsymbol{p} d \boldsymbol{q}$ of the phase space is then determined by $d N(\boldsymbol{p}, \boldsymbol{q}, t)=f(\boldsymbol{p}, \boldsymbol{q}, t) d \boldsymbol{p} d \boldsymbol{q}$. The dynamics of the distribution function follows the Boltzmann equation

$$
\frac{\partial f}{\partial t}+\dot{\boldsymbol{q}} \cdot \frac{\partial f}{\partial \boldsymbol{q}}+\dot{\boldsymbol{p}} \cdot \frac{\partial f}{\partial \boldsymbol{p}}=I=I_{\text {in }}-I_{\text {out }},
$$

where $I_{\text {in }}$ and $I_{\text {out }}$ are the so-called collision integrals. They describe how particles could change their momentum in such a way they can enter (in) or leave (out) the element of phase space surrounding $\{\boldsymbol{q}, \boldsymbol{p}\}$. Clearly, the aim of the theory is to build reliable and efficient approximation for the collision integral. A common approximation, the so-called relaxation time approximation, consists in replacing the collision integral by a linear function of $f(\boldsymbol{p}, \boldsymbol{q}, t)$ and a target distribution function $f_{0}$, leading to

$$
\frac{\partial f}{\partial t}+\dot{\boldsymbol{q}} \cdot \frac{\partial f}{\partial \boldsymbol{q}}+\dot{\boldsymbol{p}} \cdot \frac{\partial f}{\partial \boldsymbol{p}}=-\frac{f-f_{0}}{\tau},
$$

where $\tau$ is the so-called relaxation time. Indeed, $\tau$ is the time the distribution function needs to relax on the equilibrium distribution in the absence of external fields. Although rather crude, the relaxation time approximation provides excellent results in the evaluation, e.g., of the electrical conductance in mesoscopic system, via the standard Drude formula,

$$
\sigma=e^{2} n \tau / m,
$$

where $e, n$, and $m$ are the electron charge, density and mass, respectively. Clearly, here all the physical aspects of the device under investigation are related to the relaxation time and particle density. It is not difficult to see that the Boltzmann equation can be extended to study transport in periodic systems, like metal and semiconductors, as well as for phonon transport. Also, one can introduce particle-particle interaction both as part of the collisional integral and by solving the Poisson equation to obtain the electrical potential, and then the electric field, that a charge distribution exerts on a single particle. Finally, one can consider quantum effects in the collision integral thus making the theory semi-classical. Once the Boltzmann equation is solved, in linear response the thermal conductivity can be calculated as

$$
\kappa=\int d E E v \frac{\partial f}{\partial T},
$$

where $T$ is the temperature, $v$ the velocity, and $E$ the phonon energy. The definite advantage of the Boltzmann equation is that it is effectively a single particle formalism and therefore, once reliable models or approximations are available for the relaxation time, it is computationally relatively cheap to solve, even if one needs to couple it with the Poisson equation. Its disadvantages are related to its semi-classical 
nature and the difficulties in obtaining reliable and effective values for the relaxation time.

Classical approaches.-When one is interested in calculating phonon thermal transport, both the LB-DFT and the Boltzmann approaches might turn out to be inadequate. On the one hand, the LBDFT is limited to a small number of particles due to the numerical cost of solving a quantum mechanical problem. On the other hand, especially when scattering becomes dominant, the relaxation time approximation, or LB-DFT might fail in reproducing the properties of phonon transport. In these cases, recent progress has been made by classical molecular dynamics calculations to provide accurate evaluation of the thermal conductivities for fairly large systems, sizes that are not achievable with the other methods. Molecular dynamics entails solving the classical Newton equations of motion for each atom or molecule in the system taking into account the forces and position of all other particles. In this respect is therefore a full-many body approach. Approximations are needed to express the effective interaction between two atoms. This interaction is treated classically via empirical potentials that are usually parameterized starting from quantum mechanical simulations. Therefore, the quality of the results follows from the quality of the empirical potential used in the calculation. Moreover, this approach is mostly successful in the dilute limit, when one could expect that the atoms are reasonably far apart to neglect their electronic clouds to interacting strongly. Another problem currently encountered in standard molecular dynamics calculation is the introduction of proper thermostats to establish a temperature gradient in the system. Different models have been proposed with diverse degrees of success but their investigation is currently still under way, although clearly, some as the Nose-Hoover's thermostat, have established themselves as the main practical approach. Some of these limitations are overcome by considering an effective Langevin's equation for the dynamics of particles in the presence of an external random force with a colored, i.e., not white, spectrum. ${ }^{71}$ Indeed, the advantage of the colored noise lies in its ability of retaining some of the quantum effects proper of low-dimensional nanostructures. ${ }^{71,72}$ Finally, we have demonstrated that phonon can "hop" between two silica clusters: when the two clusters are close enough, the phonon wavefunctions overlap allowing for a coherent transfer of the phonon modes without any effective energy radiation. When the clusters are moved apart, the phonons can still transfer between the clusters via electromagnetic waves. ${ }^{73}$

Advances in transport theory.-The theoretical groups in the nanoTHERM consortium have focused on different aspects of thermal and electronic transport with extra care for those cases or system of relevance for the experimental part of the consortium. This has produced relevant results both at the level of fundamental theory and application to real material or devices. For the particular case of $\mathrm{Si}$ and Ge nanostructures, theory and experiment have proceeded along the same way one influencing the other, unraveling some of the physics of thermal rectification in $\mathrm{Si} / \mathrm{Ge}$ graded structures. Moving from the previous section introduction on the fundamental theories, we here explore in some details the progress made on some theoretical aspect of thermoelectric energy conversion.

Kinetic collective model.-For semiconducting materials, a common optimization strategy has been the systematic reduction of the phonon thermal conductance. For this strategy to be successful and to explain some of the recent experiments, a comprehensive theory of phonon transport has been developed. As seen, common methods are based either on the LB-DFT or on the Boltzmann equation. A key ingredient in this latter theory is the phonon relaxation time that describes how the energy is redistributed and then lost during the dynamics. An important point in kinetic-collective model is the treatment of phonon-phonon collisions. Splitting them in momentum conserving (normal) and non-conserving (umklapp), two different phonon relaxation times can be calculated, a kinetic and a collective. These two times describe how the energy is redistributed (see Figure 10).
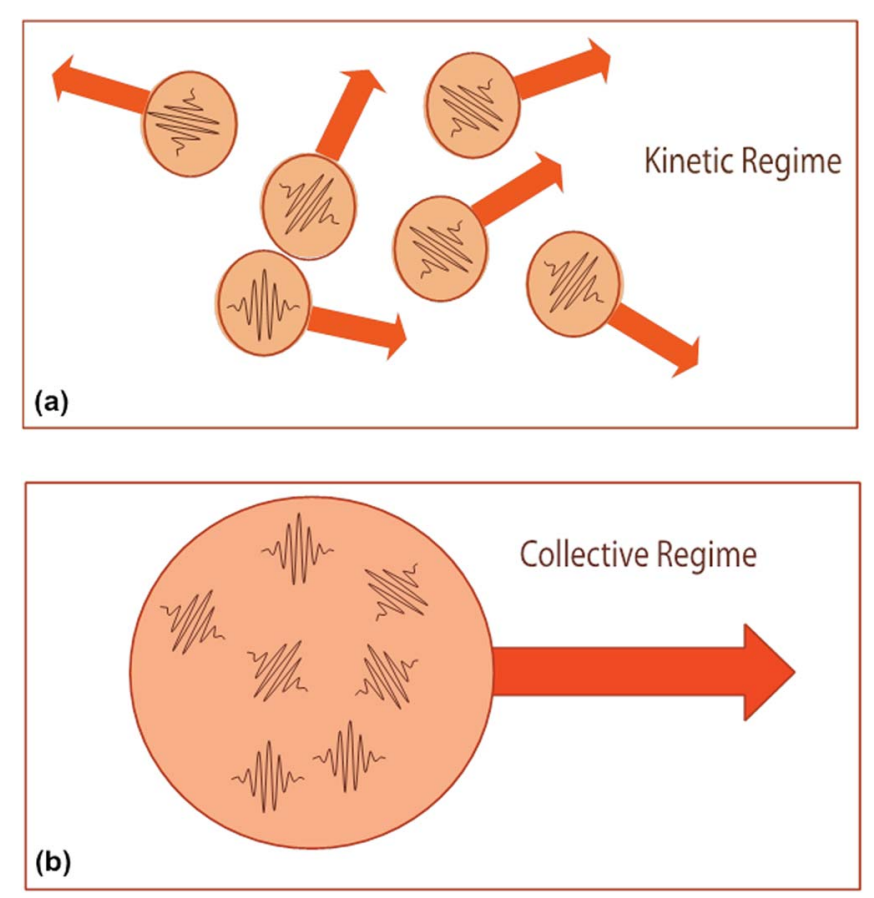

Figure 10. Kinetic (a) vs collective (b) behavior of the phonon dynamics.

We have realized that when normal collisions are not important, the transport is kinetic while when they are important, a new regime that we have called collective transport is generated. It has been shown that this collective behavior, generated by a distribution function with a nonzero momentum is fundamental to understand the thermal conductance of Si nanowires down to a few nanometers in diameter. ${ }^{74-76}$ Figure 11 summarizes our results for the wires of a few nanometers in diameter.

Further progress on the modeling has been made to bring the calculation of the relaxation time onto more solid ground. In this respect ab-initio calculation are currently performed to calculate the normal, and intrinsic relaxation times on other nanowires. Also geometrical effects are being included via an effective relaxation time introduced by scattering with the boundary of the device. Finally, as we have seen in the Graded structures section, one can attempt to reduce the thermal conductivity by introducing mass gradients in the device. We can easily consider this case by using the Boltzmann equation with a spatial dependent relaxation time for the collective motion of the phonons. $^{39}$

Seebeck coefficient for strongly correlated systems.-Disregarding its countless successes, DFT has for many years been seen only as a

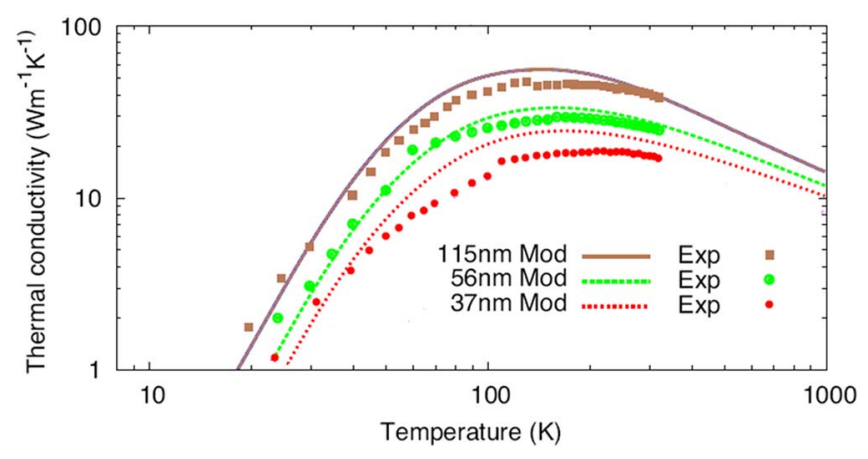

Figure 11. Thermal conductivity of Si nanowires using as fitting parameters the time relaxation of bulk $\mathrm{Si}$ (reproduced with permission from Ref. 75). 


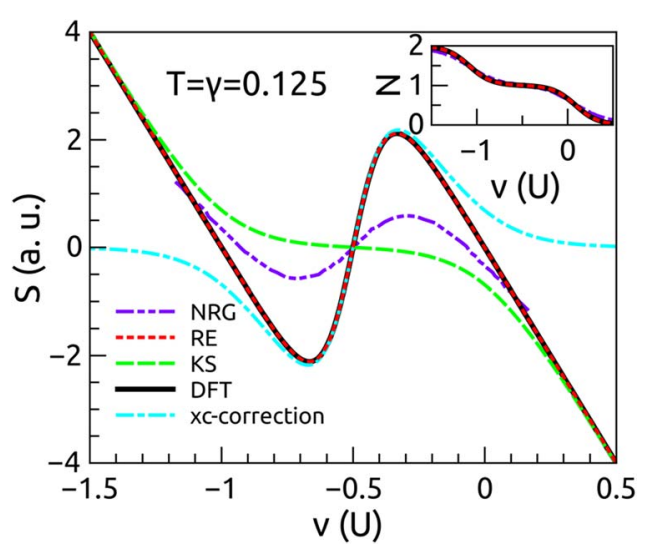

Figure 12. The Seebeck coefficient $S$ in atomic units for a single level quantum dot weakly coupled to two leads versus the gate bias (in units of U, the interaction constant). Here $\mathrm{T}$ and $\gamma$ are the working temperature and the coupling parameter between the leads and the dot, respectively.

"mean-field" theory, in the sense that it replaces the fine details of the electronic wavefunction and electronic correlation with an effective potential that contains information only about the single-particle density. Therefore, it is common belief that the theory cannot access the strong correlation regime, characteristic for example of the physics of the coulomb blockade in molecular devices. DFT is however an exact theory and as such it describes these strongly correlated regime. Recent progress has been made in this direction, and we show that strong correlation can have a dramatic effect not only on the evaluation of the electrical conductance but also on the Seebeck coefficient. The Achilles' heel of DFT is usually the lack of proper approximations to the so-called exchange and correlation (xc) energy. The problem is difficult since, although the xc energy is usually small, it is the quantity that most of the time defines the physical effects under investigation. Therefore, reliable approximations only work for a certain physical regime and experience gained in one case cannot be transferred to others. In this respect, most of the common computer packages making use of DFT rely on approximation developed for either single atoms, or the Fermi liquid. To tackle the problem of strongly correlated systems we have therefore started from a paradigmatic example, i.e., the single impurity Anderson model, and build an approximation for the $\mathrm{xc}$ potential. We have then used this approximation to evaluate the Seebeck coefficient in this case. Therefore, we have proposed a correction, completely based on DFT methods, able to amend both the electrical conductance and the Seebeck coefficient and to bring them in good agreement with other theories based on completely different approximations. ${ }^{77}$ One key ingredient that needs to be added to the picture is the so-called derivative discontinuity in the xc potential. The idea is simple: in the Coulomb-blockade regime the total energy changes abruptly when one electron is added or removed from the device. On the other hand, the nowadays-standard approximations for the $\mathrm{xc}$ potential do use only the single particle density to return the energy.

Our approach starts from some recent advancement in the direction of including this discontinuity into the xc functional. The Seebeck coefficient calculated via standard DFT $\left(S_{K S}\right)$ is therefore corrected as $S=S_{K S}+\left(\frac{\partial v_{H x c}}{\partial T}\right)_{N}$, where $V_{H x c}$ is the Hartee-xc potential for the Anderson impurity model. The simplicity of this expression is striking and its physical interpretation is clear: we need to include for a proper evaluation of the Seebeck coefficient also the xc potential difference caused by the temperature gradient.

In Figure 12, we report the Seebeck coefficient S calculated from our model for a single level quantum dot (labeled DFT), compared with the standard DFT approach (KS), the exact many-body theory (MB), and the rate equation (RE). There $\mathrm{T}$ is the working temperature and $\gamma$ is the coupling parameter between the leads and the dot. We have found impossible to compare our result with the renormalization group

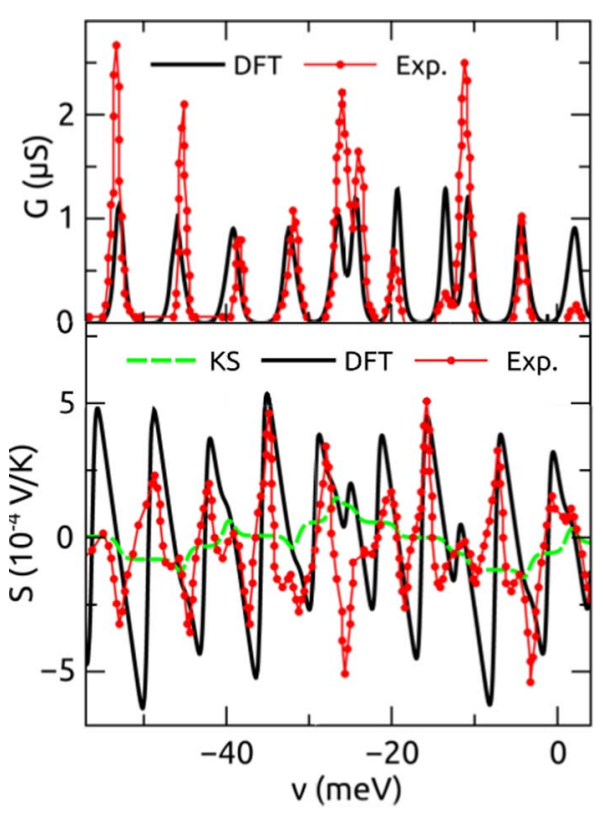

Figure 13. The electrical conductance (upper panel) and the Seebeck coefficient (lower panel) for a CNT and their comparison with experiments. Clearly our approach improves over the standard Kohn-Sham approach (reproduced with permission from Ref. 77).

approach (NRG): we believe this is due to a mismatch in the physical parameters of the two models. Looking at the xc-correction we see how important this last term is to reconstruct the correct Seebeck coefficient. Further, we have used the above correction, to evaluate the electrical conductance $G$, and the Seebeck coefficient for a CNT, as function of the bias voltage applied to the device and to compare them with available experiments. Our results are summarized in Figure 13. It is important to point out that the relevant physical pieces of information here are the peak positions, since they define the manybody energy spectrum. The peak heights (which we poorly replicate) are determined by the quality of the electrical contacts, a much difficult parameter to control experimentally and reproduce theoretically.

Open quantum system theory.-For how many positive results the LB-DFT theory can present, many attempts have been attempted to go beyond it. The reasons are clear: One may wish to not separate the different regions of the leads and the "device" as such splitting is un-natural, or we have no way to deal with real interacting electrons in the leads; or we may want to have something more than a simple featureless lead whose only function is to inject particle or energy in the system; or we may be interested also in transient phenomena. For accessing these interesting physical regimes, we need to go beyond the Landauer's picture of quantum transport and design completely novel models able to incorporate some of the effects neglected by this theory.

A way to make some progress is rooted in the theory of open quantum systems: in this framework, we study the dynamics of a system coupled to an external environment and able to exchange either particles or energy (and momentum) with it. The external environment is not necessarily uniform or stationary, but we assume it is in some sort of equilibrium. By that we mean that the total environment can be defined by a few (possibly spatially dependent) parameters, like for example a chemical potential or a temperature. For this to be true, we require that the environment be made of many degrees of freedom. We trace out those degrees of freedom and consider only they collective average behavior in the coupling with the system.

In particular, of relevance for thermal transport is the case when we consider two black body radiations as the source of the flow of energy. ${ }^{78}$ The theory allows for the description of the complete 
a)

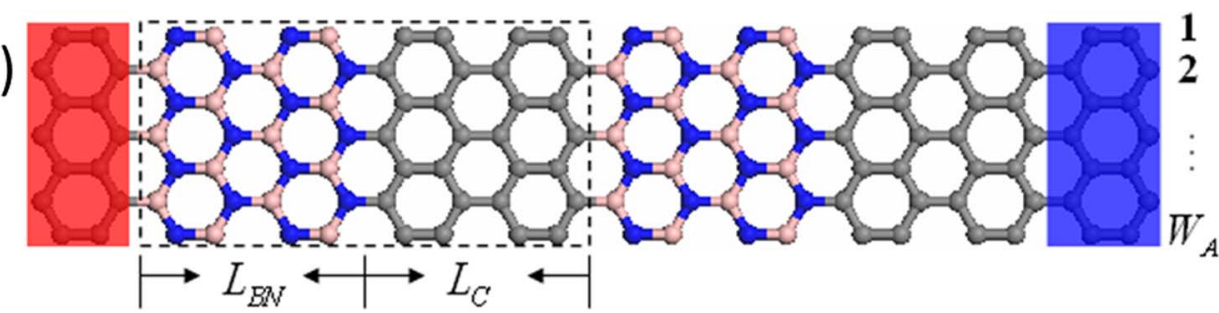

b)

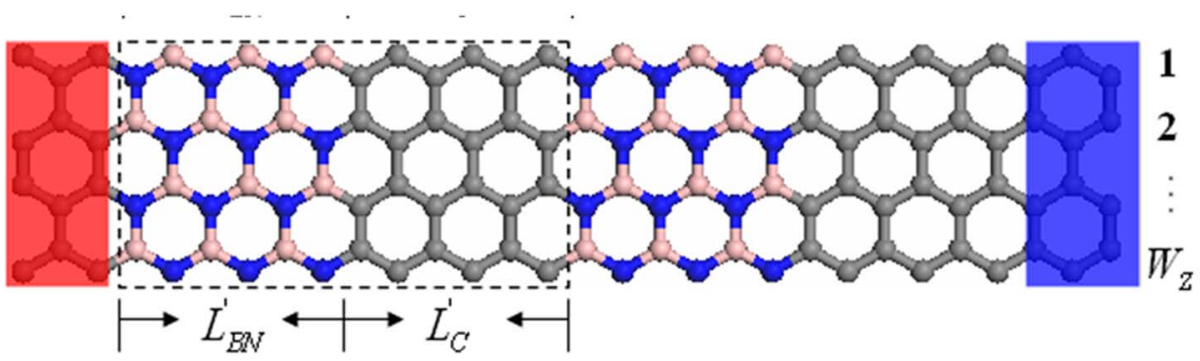

Figure 14. Graphene/BN nanoribbons with different edge terminations either zigzag or armchair, where we have identified the lengths of the BN and graphene stripes, and the total width of the nanoribbon. (Figure modified from Ref. 80).

dynamics of the system under investigation, with emphasis on the approach toward the thermal equilibrium in the long time limit. The theory is also well suited to be extended to defining a local temperature, via a local electromagnetic radiation, and it is therefore of interest in obtaining way to locally probe the heat flow. ${ }^{79}$

Theory and simulation of novel materials._Graphene and boronnitride nanoribbons.-For sure graphene needs little introduction: its special features have literally opened a new field of research on twodimensional material, proving once again that condensed matter can be a playground to discover novel and unexpected physical phenomena. Despite its exceptional electronic properties, graphene is a poor thermoelectric material. The reason for that is easily understood: the perfect crystalline structure of the monolayer allows for an easy propagation of electron and phonon, with the phonon thermal conductivity limiting drastically the figure-of-merit. Many ways have been proposed to reduce the thermal conductivity by for example introducing defects at the edges or creating holes in the material. Another possibility we have explored is the nanostructuring of graphene with other materials. In our theoretical study, we have considered boron-nitride $(\mathrm{BN})$ bidimensional structures. ${ }^{80} \mathrm{BN}$ present a honeycomb two-dimensional lattice with a periodicity matching that of graphene. As graphene, $\mathrm{BN}$ is a poor thermoelectric material. However, by nanostructuring ribbons of alternating stripes of graphene and $\mathrm{BN}$, we have been able to contain the increase in the thermal conductivity with size thus achieving a strong enhancement of the overall figure-of-merit. In Figure 14 we report some of the different nanoribbons considered. Each nanoribbon is identified by its edge terminations (either zigzag or armchair), by the relative length of the $\mathrm{BN}$ and graphene stripes, and by their total width. The lengths of the stripes can be different. We found, via numerical calculations that the most promising material for thermoelectric material would be a quite narrow armchair nanoribbon with equal length alternating stripes of carbon and BN. This can be seen in Figure 15 where we report the increase of the figure of merit $(\boldsymbol{Z T})$, with respect to the pristine graphene $\left(\boldsymbol{Z} \boldsymbol{T}_{\mathbf{0}}\right)$ as function of the ratio of the lengths of the $\mathrm{C}$ and $\mathrm{BN}$ stripes, $\gamma$, for different lengths of the total unit cell $\left(\boldsymbol{L}_{s}=\boldsymbol{L}_{\boldsymbol{C}}+\boldsymbol{L}_{B N}\right)$.

As we clearly see from Figure 15, we can draw a first lesson from theory: nanostructuring two poor thermoelectric materials, we can in principle increase the overall efficiency by a factor larger than 10 . This has been one of the common research paths we have followed in our work.
Single layer silicon and germanium.-Graphene has the potential to change our view of electronic devices, but admittedly it is a difficult material to work with, especially when one tries to integrate it with the existing technology. Also, to make it useful for technological applications, one need to open up a finite gap at the Fermi energy, via for example doping, which however can degrade its other superior qualities. At the same time, looking at the periodic table, one can wonder if Silicon and Germanium, the prominent materials in our nowadays electronic technology can have a two-dimensional phase similar to Carbon. The answer is in the positive, and recently silicene and germanene two bi-dimensional allotropes of Silicon and Germanium have been produced and studied, after being predicted theoretically, ${ }^{81}$ although the actual atomic structure of these allotropes is not completely clarified, since they have been synthetized on a metallic substrate (Ag). Indeed, silicene and germanene have been predicted to be buckled structures, where the $\mathrm{Si}$ or $\mathrm{Ge}$ atoms do adjust on two different planes. In Figure 16, we show the atomic position of silicene and germanene, with a side-view showing the buckling and their electronic band structures. Notice that, according to our calculations, buckling destroys the Dirac points, but especially in silicene opens up a finite gap that

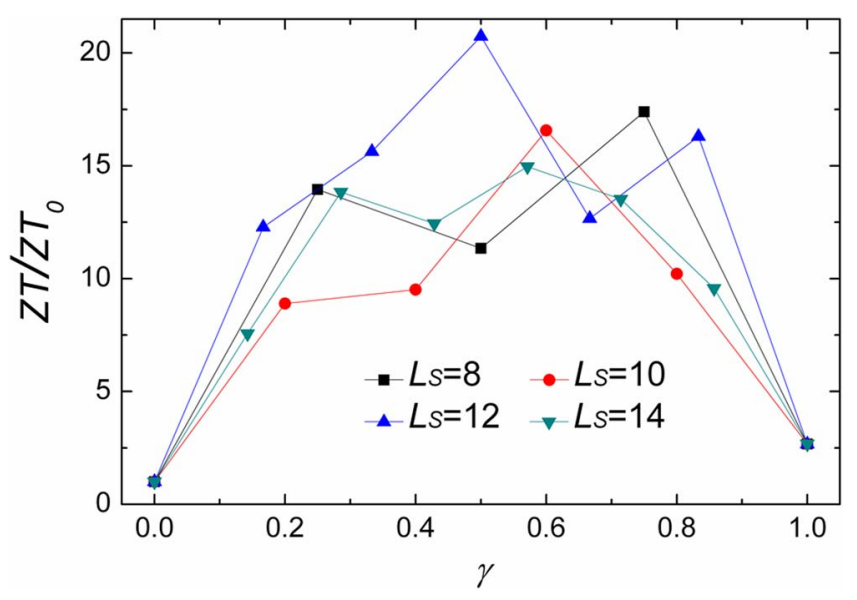

Figure 15. The relative increase of the figure of merit in a graphene/BN armchair nanoribbon as a function of the relative length of the stripes (reproduced with permission from Ref. 80). 
(a)

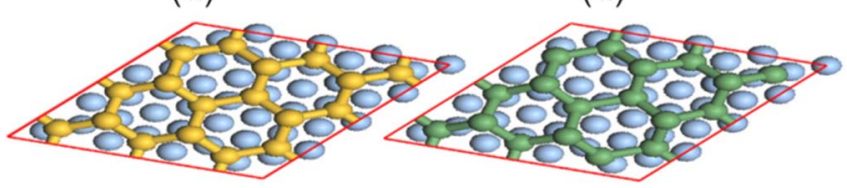

(b)

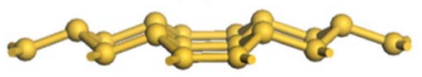

(c)

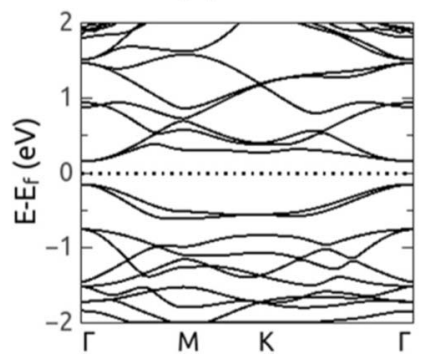

(e)

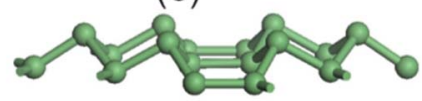

(f)

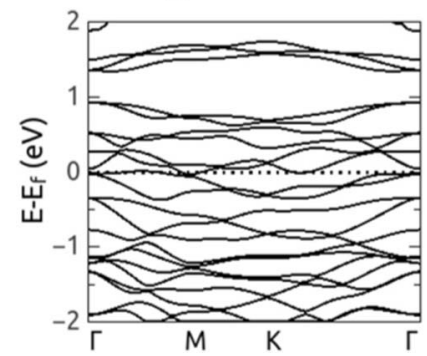

Figure 16. Atomic positions (a and d), buckling (b and e), and electronic structure (c and f) of silicene (yellow) and germanene (green) 2D materials. Notice the bandgap of silicene at the Fermi level (reproduced with permission from Ref. 81)

could be useful for technological applications. ${ }^{81}$ Germanene on the other hand, turns out to be a metal. The electronic band structures are obtained without considering the presence of the Ag substrate. According to our calculations, silicene and germanene would be poor thermoelectric materials due to a small power factor and large thermal conductance. Again, we have tried to reduce the dimensionality of the system, by considering nanoribbons of $\mathrm{Si}$ and $\mathrm{Ge}$, and by nanostructuring them. In the first case, we have obtained a quite promising increase of the overall figure of merit, with a peak $Z T=1$, or 1.5 , for both systems, with Germanene armchair nanoribbons the best material in principle. Similarly to what we have done for the graphene/BN structures we have considered alternating stripes of $\mathrm{Si}$ and Ge. Contrary to our finding for graphene, the overall figure of merit of $\mathrm{Si}$ and Ge nanostructures does not increase significantly with respect to the pure $\mathrm{Si}$ and $\mathrm{Ge}$ nanoribbons, since we have reached a peak value of $Z T=2.5$, (see Ref. 81) much less than the 10-fold increase observed in the graphene/BN nanoribbons. While this value would be good for technological applications, we believe it will be reduced in real device larger than what we can investigate in-silico, since the phonon transport increase fast with the width of the nanoribbons. A reason for the lack of improvement is probably related to a reduced impact on the phonon thermal conductance in the $\mathrm{Si} / \mathrm{Ge}$ nanoribbons. Indeed, for the unit cells we can investigate, phonon trapping and scattering are effective only for high-energy phonons usually less responsible for heat transport. To affect the low frequency phonons, we would need to consider larger super-cells, a task unattainable with our actual numerical resources.

Silicon and germanium nanowires.-Bulk $\mathrm{Si}$ is a bad thermoelectric material, with a figure of merit of about 0.01. As suggested in the past, nanostructuring can improve the figure of merit dramatically. Indeed, it has been shown experimentally that $\mathrm{Si}$ nanowires, with rough surfaces, can achieve a figure of merit, $Z T$, of about 0.5 . This remarkable result has been attributed to the dramatic decrease in the thermal conductance due to the increase phonon scattering by the rough surfaces. ${ }^{25,59,82-84}$

In the quest for efficient thermoelectric materials, we have proposed a novel approach to improve the overall figure of merit of $\mathrm{Si}$ nanowires. Our calculations show that covering the Si nanowires with a thin shell of $\mathrm{Ge}$ (or an alloy of $\mathrm{Si}$ and $\mathrm{Ge}$ ) does improve the figure

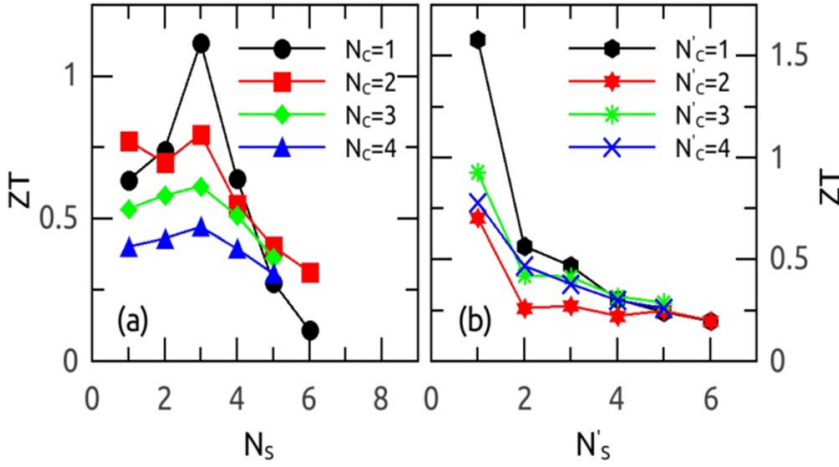

Figure 17. Thermoelectric figure of merit as a function of the number of atomic layers in the shell for (a) a clean $\mathrm{Si} / \mathrm{Ge}$ and (b) a clean $\mathrm{Ge} / \mathrm{Si}$ nanowire (reproduced with permission from Ref. 85).

of merit. In particular, there is an optimal radius of the shell that corresponds to about 3 atomic layers of Ge. At the same time, we have considered $\mathrm{Ge}$ nanowires covered with a thin shell of Si. In this case, we observe that $Z T$ is a monotonous decreasing function of the shell radius. We can explain this effect by observing that the presence of the thin shell creates an interface for the phonons that induces, especially for the phonons propagating along the radius of the nanowire, back-scattering and interference effects. The phonon thermal conductance therefore does not increase as fast as adding layers of Si to the nanowire. At the same time, the electrical conductance increases since the presence of the shell opens up new channels for electrical propagation. Especially for a Si core/Ge shell nanowire, the figure of merit reaches therefore its maximum due to the competition between the electrical and thermal conductances, while the Seebeck coefficient stays essentially constant. ${ }^{85}$

Let us begin with considering a thin $\mathrm{Si}$ or Ge nanowire grown in the (111) direction. We further cover the nanowire with a thin shell made of a few atomic layers of either $\mathrm{Ge}$ or $\mathrm{Si}$ or an alloy of them. Our results for the $\mathrm{Si}$ core / Ge shell and Ge core/Si shell nanowires figure-of-merit are presented in Figure 17. For the Si core/Ge shell nanowire, we clearly observe a dramatic increase of $Z T$ at around three atomic layers in the shell.

We have furthermore observed that there is an optimal working temperature that provides the highest possible figure of merit. Clearly, one would like to identify such an optimal working condition from the outset before performing any actual experiment. Indeed, this would provide an important help in designing high efficient devices. For our $\mathrm{Si} / \mathrm{Ge}$ nanowires, we have observed a linear relation between the electron energy gap of the wire, $E_{g}$, and the temperature at which the figure of merit is maximal, $T_{C}$. We report this behavior in Figure 18 , in agreement with the linear behavior between $E_{g}$ and $T_{C}$ of the Sharp-Goldsmid's formula. ${ }^{86}$

Polymeric materials.-Polymers attract an increasing amount of scientific interest due to their potential diversity of applications, their relative small cost, and adaptability to different external conditions. For this reason the recent discovery and synthesis of semiconducting polymers are seen as a major scientific breakthrough and have triggered a few works on the potential of polymers for thermoelectric applications. While such an interested existed well before the starting of our consortium, the figure-of-merit of those materials was too small for any possible applications. But with these novel materials, the figure of merit we have achieved becomes almost competitive, of the order of 0.2 and comparable with the state-of-the-art at $0.42:{ }^{87}$ still small but with the advantage of working with polymers, for a series of potential applications like wearable electronics that is not achievable with other inorganic materials. Also to further increase the range of potential applications, hybrid organic/inorganic devices can be built which share the advantages of both worlds, i.e., good electrical and reduced thermal transport. 


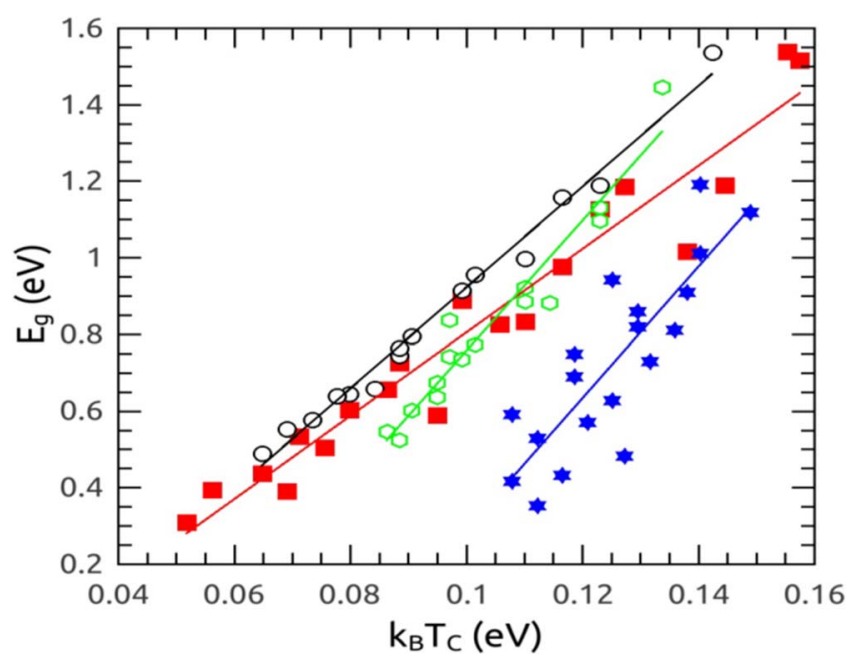

Figure 18. The electron energy bandgap $\mathrm{E}_{\mathrm{g}}$ versus $\mathrm{k}_{\mathrm{B}} \mathrm{T}_{\mathrm{C}}$ for clean $\mathrm{Si} / \mathrm{Ge}$ (rectangle) and $\mathrm{Ge} / \mathrm{Si} \mathrm{NWs}$ (star), Si/disordered SiGe (ellipse) and Ge/disordered SiGe NWs (hexagon). The red, blue, black and green lines are the linearly fitted curves (reproduced with permission from Ref. 85).

Modeling transport in polymers is difficult since the standard method work mostly for delocalized systems, while in polymers electrons and phonons are likely strongly localized in the chain. At the same time, doping necessary to inject electrons in the conductance band and increase transport, does usually have an effect also on the polymeric chain structure, and not considering such effects might be detrimental for understanding the physics of the device. Finally, although we can think of them as quite large strand, polymers are rarely periodic. Therefore a brute force ab-initio calculation should consider a large enough number of basic unit cells in order to capture some of the folding. For these reasons, the models developed in the consortium to understand the properties of polymeric devices are mostly phenomenological and based essentially on some approximation on how the electrons and phonons hop through strands of the chain. Moreover, for a complete description of a polymeric strand, we need to consider also its folding state: it has been show for example that partial un-folding or straightening of part of the chain can increase electrical transport.

On the other hand, some semi-empirical models can provide insight into some aspects of the energy and electrical transport in polymer based devices. This is the case for example of the P3HT polymer mixed with both doped and un-doped CNTs. Indeed, the observed behaviors can be fitted with a simple percolation model, where both the Seebeck coefficient and the electrical conductance follow power laws, $\sigma=$ $\sigma_{C N T}\left|c-c^{*}\right|^{p}, S=S_{C N T}\left|c-c^{*}\right|^{p}$ where $c$ is the actual concentration of CNT, $c^{*}$ is the minimum percolation concentration, and $p$ equals $4 / 3$ in $2 \mathrm{D}$ and 2 in $3 \mathrm{D}$ is a percolation parameter. The parameters $c^{*}, \sigma_{C N T}$, and $S_{C N T}$ can be determined from the experimental data or could be evaluated from a fundamental model of the CNT. The physical picture one can extract from the experiments is that the CNT construct a network for the electron to percolate through by hopping from the CNT to the P3HT: a small CNT concentration does not allow for such a network to form, and the electrical conductance and Seebeck coefficients are strongly suppressed.

\section{Summary}

In this review article have shown most of the joint project "Tailoring Electronic and Phononic Properties of Nanomaterials: Towards Improved Thermoelectricity (nanoTHERM)", a Spanish research Consolider project focused on the understanding of the thermoelectric materials and their tailoring of both their electronic and phononic properties toward the optimization of thermoelectric devices for low and high temperature regimes.
The initial purpose of the project was to answer some of the open scientific questions that arise from trying to improve thermoelectric materials. On the one hand, in the case of organic materials, the understanding of the electrical conductivity mechanisms and finding ways of increasing it, which is the main drawback their present for practical applications was pursued. Achievements such as figures of merit of 0.22 in PEDOT, the obtention of both $\mathrm{p}$ - and n-type P3HT polymers in such a way that they can be integrated in a device, or the increase of the power factor in PANI of three orders of magnitude with respect to the bare polymer thanks to the mixing with GNPs are some of the results obtained. On the other hand, for inorganic materials, the effect of nanostructuring, which opens the possibility of decouple thermal and electrical properties, was studied. With this approach, a reduction in the thermal conductivity of bismuth telluride with narrower nanowire diameter from the bulk value of $2.2 \mathrm{~W} \cdot \mathrm{m}^{-1} \cdot \mathrm{K}^{-1}$ to 0.5 $\mathrm{W} \cdot \mathrm{m}^{-1} \cdot \mathrm{W}^{-1}$ in $30 \mathrm{~nm}$ diameter nanowires has been measured and understood. Moreover, the effect of phononic crystals on the thermal conductivity has been studied in depth and actual devices, such as a micro thermoelectric generator based on silicon thin membranes, have been developed.

\section{Acknowledgments}

The authors are grateful to the Spanish MEC for the financial support through the CONSOLIDER project CSD2010-00044. They would like to specially thank all the members of the consortium that have contributed to the work here presented under the direction of Prof. Dr. Clivia M. Sotomayor Torres.

\section{References}

1. M. Culebras, C. M. Gómez, and A. Cantarero, Materials, 7, 6701 (2014).

2. C. M. Gómez, M. Culebras, A. Cantarero, B. Redondo-Foj, P. Ortiz-Serna, M. Carsí, and M. J. Sanchis, Applied Surface Science, 275, 295 (2013).

3. B. Dörling, V. Vohra, T. T. Dao, M. Garriga, H. Murata, and M. Campoy-Quiles, Journal of Materials Chemistry C, 2, 3303 (2014).

4. M. Culebras, C. Gomez, and A. Cantarero, Journal of Materials Chemistry A, 2, 10109 (2014)

5. J. Martín, M. Campoy-Quiles, A. Nogales, M. Garriga, M. I. Alonso, A. R. Goñi, and M. Martín-González, Soft matter, 10, 3335 (2014).

6. B. Redondo-Foj, P. Ortiz-Serna, M. Carsí, M. J. Sanchis, M. Culebras, C. M. Gómez, and A. Cantarero, Polymer International, 64, 284 (2015).

7. M. Culebras, R. Torán, C. M. Gómez, and A. Cantarero, Nanoscale Research Letters, 9, 1 (2014).

8. D. Nassyrov, C. Müller, A. Roigé, I. Burgués-Ceballos, J. O. Ossó, D. B. Amabilino, M. Garriga, M. I. Alonso, A. R. Goñi, and M. Campoy-Quiles, Journal of Materials Chemistry, 22, 4519 (2012)

9. C. Bounioux, P. Díaz-Chao, M. Campoy-Quiles, M. S. Martín-González, A. R. Goñi, R. Yerushalmi-Rozen, and C. Müller, Energy \& Environmental Science, 6, 918 (2013).

10. M. Culebras, A. Madroñero, C. Mota, C. Gómez, J. M. Amo, and A. Cantarero, Radiation Effects and Defects in Solids, 169, 620 (2014).

11. A. Madroñero, J. Robla, J. García-Hierro, M. S. Martín-González, P. Díaz, and A. López, Journal of Materials Science: Materials in Electronics, 23, 1470 (2012).

12. M. Culebras, A. Madroñero, A. Cantarero, J. M. Amo, C. Domingo, and A. López, Nanoscale Research Letters, 7, 1 (2012).

13. C. Mota, M. Culebras, A. Cantarero, A. Madro, C. M. Gómez, J. M. Amo, and J. I. Robla, Advances in Materials Physics and Chemistry, 3, 153 (2013).

14. B. Abad, I. Alda, P. Díaz-Chao, H. Kawakami, A. Almarza, D. Amantia, D. Gutierrez, L. Aubouy, and M. Martín-González, Journal of Materials Chemistry A, 1, 10450 (2013).

15. J. Maiz, M. M. Rojo, B. Abad, A. A. Wilson, A. Nogales, D.-A. Borca-Tasciuc, T. Borca-Tasciuc, and M. Martín-González, RSC Advances, 5, 66687 (2015).

16. M. Culebras, C. M. Gómez, and A. Cantarero, Journal of Materials Science, 48, 2855 (2013).

17. M. Culebras, B. Uriol, C. M. Gómez, and A. Cantarero, Physical Chemistry Chemical Physics, 17, 15140 (2015).

18. M. Culebras, A. M. López, C. M. Gómez, and A. Cantarero, Sensors and Actuators A: Physical, 239, 161 (2016).

19. A. A. Guilbert, M. Schmidt, A. Bruno, J. Yao, S. King, S. M. Tuladhar, T. Kirchartz, M. I. Alonso, A. R. Goñi, and N. Stingelin, Advanced Functional Materials, 24, 6972 (2014).

20. B. Dörling, J. D. Ryan, J. D. Craddock, A. Sorrentino, A. E. Basaty, A. Gomez, M. Garriga, E. Pereiro, J. E. Anthony, M. C. Weisenberger, A. R. Goñi, C. Müller, and M. Campoy-Quiles, Adv Mater, 28, 2782 (2016).

21. M. M. Rojo, J. Martín, S. Grauby, T. Borca-Tasciuc, S. Dilhaire, and M. Martin-Gonzalez, Nanoscale, 6, 7858 (2014).

22. Thermoelectric Bi2Te3 nanomaterials, Wiley-VCH, Weinheim (2015). 
23. J. A. Perez-Taborda, M. M. Rojo, J. Maiz, N. Neophytou, and M. Martin-Gonzalez, Scientific Reports, 6 (2016).

24. O. Korotchenkov, A. Nadtochiy, V. Kuryliuk, C. Wang, P. Li, and A. Cantarero, European Physical Journal B, 87, 64 (2014).

25. A. Sellitto, F. Alvarez, and D. Jou, Journal of Applied Physics, 109, 064317 (2011)

26. O. Caballero-Calero and M. Martín-González, Scripta Materialia, 111, 54 (2016).

27. B. Graczykowski, M. Sledzinska, F. Alzina, J. Gomis-Bresco, J. Reparaz, M. Wagner, and C. M. Sotomayor Torres, Physical Review B, 91, 075414 (2015).

28. A. Sellitto, D. Jou, and J. Bafaluy, Proceedings of the Royal Society A-Mathematical Physical and Engineering Sciences, 468, 1217 (2012).

29. A. Pérez del Pino, E. György, I. Marcus, J. Roqueta, and M. Alonso, Nanotechnology, 22, 295304 (2011).

30. J. Reparaz, A. Goñi, M. Alonso, and M. Garriga, physica status solidi (b), 250, 760 (2013).

31. V. Kuryliuk, O. Korotchenkov, and A. Cantarero, Physical Review B, 85, 075406 (2012).

32. C. V. Manzano, B. Abad, M. M. Rojo, Y. R. Koh, S. L. Hodson, A. M. L. Martinez, X. Xu, A. Shakouri, T. D. Sands, T. Borca-Tasciuc, and M. S. Martín-González, Scientific Reports, 6, 19129 (2016).

33. O. Caballero-Calero, P. Díaz-Chao, B. Abad, C. V. Manzano, M. D. Ynsa, J. J. Romero, M. M. Rojo, and M. S. Martín-González, Electrochimica Acta, 123 117 (2014)

34. O. Caballero-Calero, M. Mohner, M. Casas, B. Abad, M. Rull, D. A. Borca-Tasciuc, and M. Martín-González, Materials Today: Proceedings, 2, 620 (2015).

35. C. Rodríguez-Fernández, C. V. Manzano, A. H. Romero, J. Martín, M. Martín-González, M. M. de Lima Jr, and A. Cantarero, Nanotechnology, 27, 075706 (2016)

36. J. Martín, C. V. Manzano, O. Caballero-Calero, and M. Martín-GonzaF́lez, ACS applied materials \& interfaces, 5, 72 (2012).

37. M. Muñoz Rojo, S. Grauby, J.-M. Rampnoux, O. Caballero-Calero, M. Martin-Gonzalez, and S. Dilhaire, Journal of Applied Physics, 113, 054308 (2013).

38. J. Reparaz, I. Marcus, A. Goñi, M. Garriga, and M. Alonso, Journal of Applied Physics, 112, 023512 (2012)

39. P. Ferrando-Villalba, A. F. Lopeandía, F. X. Alvarez, B. Paul, C. de Tomás, M. I. Alonso, M. Garriga, A. R. Goñi, J. Santiso, and G. Garcia, Nano Research, 8, 2833 (2015)

40. E. Chávez, J. Cuffe, F. Alzina, and C. Sotomayor Torres, in Journal of Physics: Conference Series, 395, 012105 (2012).

41. E. Chávez-Ángel, J. Reparaz, J. Gomis-Bresco, M. Wagner, J. Cuffe, B. Graczykowski, A. Shchepetov, H. Jiang, M. Prunnila, J. Ahopelto, F. Alzina, and C. M. Sotomayor Torres, APL Materials, 2, 012113 (2014).

42. S. Neogi, J. S. Reparaz, L. F. C. Pereira, B. Graczykowski, M. R. Wagner, M. Sledzinska, A. Shchepetov, M. Prunnila, J. Ahopelto, and C. M. Sotomayor-Torres, ACS Nano, 9, 3820 (2015)

43. P. Ferrando-Villalba, A. Lopeandia, L. Abad, J. Llobet, M. Molina-Ruiz, G. Garcia, M. Gerbolès, F. Alvarez, A. Goñi, and F. Muñoz-Pascual, Nanotechnology, 25, 185402 (2014)

44. A. Perez-Marín, A. Lopeandía, L. Abad, P. Ferrando-Villaba, G. Garcia, A. Lopez, F. Muñoz-Pascual, and J. Rodríguez-Viejo, Nano Energy, 4, 73 (2014).

45. J. Cuffe, O. Ristow, E. Chávez, A. Shchepetov, P.-O. Chapuis, F. Alzina, M. Hettich, M. Prunnila, J. Ahopelto, T. Dekorsy, and C. M. Sotomayor Torres, Physical Review Letters, 110, 095503 (2013).

46. B. Graczykowski, M. Sledzinska, N. Kehagias, F. Alzina, J. Reparaz, and C. M. Sotomayor Torres, Applied Physics Letters, 104, 123108 (2014).

47. M. Sledzinska, B. Graczykowski, F. Alzina, J. Lopez, S., and C. Sotomayor Torres, Microelectronic EngineeringMicroelectronic Engineering, 149, 41 (2016).

48. B. Graczykowski, A. El Sachat, J. S. Reparaz, M. Sledzinska, M. R. Wagner, E. Chavez-Angel, S. Volz, Y. Wu, F. Alzina, and C. M. Sotomayor Torres, under review (2017).

49. M. R. Wagner, B. Graczykowski, J. S. Reparaz, A. El Sachat, M. Sledzinska F. Alzina, and C. M. Sotomayor Torres, Nano Letters, 16, 5661 (2016).

50. A. Sellitto, D. Jou, and V. Cimmelli, Acta applicandae mathematicae, 122, 435 (2012).

51. M. Criado-Sancho, L. Del Castillo, J. Casas-Vázquez, and D. Jou, Physics Letters A 376, 1641 (2012)

52. M. Criado-Sancho, F. Alvarez, and D. Jou, Journal of Applied Physics, 114, 053512 (2013)
53. A. Santander-Syro, O Copie, T. Kondo, F. Fortuna, S. Pailhes, R. Weht, X. Qiu, F. Bertran, A. Nicolaou, and A. Taleb-Ibrahimi, Nature, 469, 189 (2011).

54. S. Ohta, H. Ohta, and K. Koumoto, Journal of the Ceramic Society of Japan, 114 $102(2006)$

55. S. Bhansali, PhD thesis submitted to the Universidad Autonoma de Barcelona (December 2016) and manuscripts in preparation.

56. P. Tomes, C. Suter, M. Trottmann, A. Steinfeld, and A. Weidenkaff, Journal of Materials Research, 26, 1975 (2011).

57. L. Bocher, M. Aguirre, D. Logvinovich, A. Shkabko, R. Robert, M. Trottmann, and A. Weidenkaff, Inorganic Chemistry, 47, 8077 (2008).

58. H. Ohta, S. Kim, Y. Mune, T. Mizoguchi, K. Nomura, S. Ohta, T. Nomura, Y. Nakanishi, Y. Ikuhara, M. Hirano, H. Hosono, and K. Koumoto, Nature Mate rials, 6, 129 (2007).

59. A. Sellitto, F. Alvarez, and D. Jou, International Journal of Heat and Mass Transfer, 55, 3114 (2012)

60. M. Muñoz Rojo, O. Calero Caballero, A. Lopeandia, J. Rodriguez-Viejo, and M. Martín-Gonzalez, Nanoscale, 5, 11526 (2013).

61. M. Culebras, C. Gomez, A. Gomez, F. Sapina, and A. Cantarero, Journal of Electronic Engineering, 2, 59 (2014).

62. I. C. Marcus, I. Berbezier, A. Ronda, M. I. Alonso, M. Garriga, A. R. Goni, E. Gomes, L. Favre, A. Delobbe, and P. Sudraud, Crystal Growth \& Design, 11, 3190 (2011).

63. J. Reparaz, N. Peica, R. Kirste, A. Goni, M. Wagner, G. Callsen, M. Alonso, M. Garriga, I. Marcus, and A. Ronda, Nanotechnology, 24, 185704 (2013).

64. J. Reparaz, G. Callsen, M. Wagner, F. Güell, J. R. Morante, C. M. Sotomayor Torres, and A. Hoffmann, APL Materials, 1, 012103 (2013).

65. A. Goñi, L. Muniz, J. Reparaz, M. Alonso, M. Garriga, A. Lopeandia, J. Rodríguez-Viejo, J. Arbiol, and R. Rurali, Physical Review B, 89, 045428 (2014).

66. J. Loureiro, J. R. Santos, A. Nogueira, F. Wyczisk, L. Divay, S. Reparaz, F. Alzina C. M. Sotomayor Torres, J. Cuffe, and F. Montemor, Journal of Materials Chemistry A, 2, 6456 (2014)

67. J. Loureiro, N. Neves, R. Barros, T. Mateus, R. Santos, S. Filonovich, S. Reparaz, C. M. Sotomayor Torres, F. Wyczisk, and L. Divay, Journal of Materials Chemistry A, 2, 6649 (2014)

68. J. Cuffe, E. ChaF́vez, A. Shchepetov, P.-O. Chapuis, E. H. El Boudouti, F. Alzina, T. Kehoe, J. Gomis-Bresco, D. Dudek, Y. Pennec, B. Djafari-Rouhani, M. Prunnila, J. Ahopelto, and C. M. Sotomayor Torres, Nano Letters, 12, 3569 (2012).

69. M. Di Ventra, Electrical Transport in Nanoscale Systems, Cambridge University Press, New York (2008).

70. R. D'Agosta, Physical Chemistry Chemical Physics, 15, 1758 (2013).

71. A. Savin, Y. Kosevich, and A. Cantarero, Physical Review B, 86, 064305 (2012)

72. Y. Kosevich, A. V. Savin, and A. Cantarero, Nanoscale Research Letters, 8, 7 (2013).

73. S. Xiong, K. Yang, Y. Kosevich, Y. Chalopin, R. D'Agosta, P. Cortona, and S. Volz, Physical Review Letters, 112, 114301 (2014)

74. C. de Tomas, A. Cantarero, A. Lopeandia, and F. Alvarez, Journal of Applied Physics, 115 (2014).

75. C. de Tomas, A. Cantarero, A. Lopeandia, and F. Alvarez, Proceedings of the Royal Society A-Mathematical Physical and Engineering Sciences, 470 (2014).

76. C. de Tomas, A. Cantarero, A. Lopeandia, and F. Alvarez, Journal of Applied Physics, 118 (2015)

77. K. Yang, E. Perfetto, S. Kurth, G. Stefanucci, and R. D’Agosta, Physical Review B, 94, 081410 (2016)

78. R. Biele, R. D'Agosta, and A. Rubio, Physical Review Letters, 115, 056801 (2015).

79. R. Biele and R. D'Agosta, Journal of Physics: Condensed Matter, 24, 273201 (2012)

80. K. Yang, Y. Chen, R. D'Agosta, Y. Xie, J. Zhong, and A. Rubio, Physical Review B, 86, 045425 (2012)

81. K. Yang, S. Cahangirov, A. Cantarero, A. Rubio, and R. D'Agosta, Physical Review $B, \mathbf{8 9}, 125403$ (2014).

82. A. Sellitto, V. Cimmelli, and D. Jou, International Journal of Heat and Mass Transfer, 57, 109 (2013).

83. F. Alvarez, D. Jou, and A. Sellitto, Journal of Heat Transfer-Transactions of the Asme, 133, 022402 (2011)

84. D. Jou, A. Sellitto, and F. Alvarez, Proceedings of the Royal Society A-Mathematical Physical and Engineering Sciences, 467, 2520 (2011)

85. K. Yang, A. Cantarero, A. Rubio, and R. D'Agosta, Nano Research, 8, 2611 (2015).

86. H. J. Goldsmid, Introduction to Thermoelectricity, New York (2010).

87. G.-H. Kim, L. Shao, K. Zhang, and K. P. Pipe, Nature Materials, 12, 719 (2013) 


\title{
Erratum: Review-Towards the Next Generation of Thermoelectric Materials: Tailoring Electronic and Phononic Properties of Nanomaterials [ECS J. Solid State Sci. Technol., 6, N3065 (2017)]
}

\author{
Olga Caballero-Calero ${ }^{1}$ and Roberto D'Agosta ${ }^{2,3}$ \\ ${ }^{I}$ IMM-Instituto de Microelectrónica de Madrid (CNM-CSIC), E-28760 Tres Cantos, Madrid, Spain \\ ${ }^{2}$ Nano-Bio Spectroscopy Group and ETSF, Universidad del País Vasco, CFM CSIC-UPV/EHU, \\ 20018 San Sebastián, Spain \\ ${ }^{3}$ IKERBASQUE, Basque Foundation for Science, E-48013 Bilbao, Spain
}

(C) 2018 The Electrochemical Society. [DOI: 10.1149/2.0041804jss] Published March 16, 2018.

On page N3066, in the left-hand column, the fourth sentence in the third full paragraph should read:

In order to increase the electrical conductivity of these nanocomposites, electrical conductive fillers GNP were also introduced in the composite, obtaining power factors of $14 \mu \mathrm{W} \cdot \mathrm{m}^{-1} \cdot \mathrm{K}^{-2}$ when $50 \%$ wt GNPs were added, ${ }^{14}$ which is three orders of magnitude with respect to PANI itself (see Fig. 1, where the effect of the charge carriers in the electrical conductivity is shown
(Fig. 1a) while the thermal conductivity (Fig. 1b) is maintained low).

On page N3066, in the right-hand column, the second sentence in the second full paragraph should read:

The composite formed with PEDOT:PSS and different contents of graphite was studied as thermoelectric material for room-temperature applications, showing power factors over $5 \mu \mathrm{W} \cdot \mathrm{m}^{-1} \cdot \mathrm{K}^{-2}$ for composites with a $80 \% \mathrm{wt}$ of expanded graphite. ${ }^{16}$ 